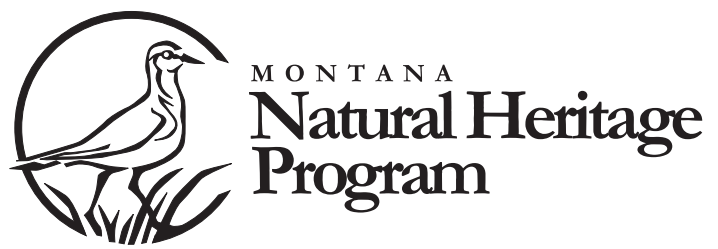

A program of the Natural

Resource Information System, Montana State Library

\section{Plant Species of Concern}

April 2003

\title{
Introduction
}

The Montana Natural Heritage Program (MTNHP) serves as the state's clearinghouse and principle information source on Species of Concern - plants and animals that are at risk or potentially at risk in Montana. This report, which updates and replaces our 2001 publication, identifies 330 vascular plant Species of Concern, and 220 vascular plants of Potential Concern in the state, based on information gathered from field inventories, publications and reports, herbarium specimens, and the knowledge of Montana botanists. Also included are the preliminary status ranks for 111 bryophyte species and 114 lichen species currently considered rare or potentially rare in Montana, based on recent work by experts studying these groups; that listing remains unchanged from the 2001 publication.

This update includes one new Species of Concern. Seven have been downgraded to Potential Concern (see page 2); two other species were added as Potential Concern, bringing the total additions to nine. Also, as of this update, state ranks are now consistent with the lists; all Species of Concern have ranks of S1 through S2S3 or SH (historical records only), whereas the Potential Concern list includes those ranked as $\mathrm{S} 3$ or lower or of uncertain status.

The Montana Natural Heritage Program continuously reviews and updates status ranks as new information and insights are generated by field surveys, research, and observations. Proposed rank or rank changes, and information supporting them, are informally reviewed by botanists and resource specialists throughout the state, who provide their perspectives and any additional information to guide the biological assessment process. The information that we receive from botanists and others throughout the state is essential in this process, and contributes to more accurate assessments of species' status. We continue to ask that all observations of these species be reported to the Heritage Program, with as much detail as possible. Field survey forms specifying the information that should be submitted are available on

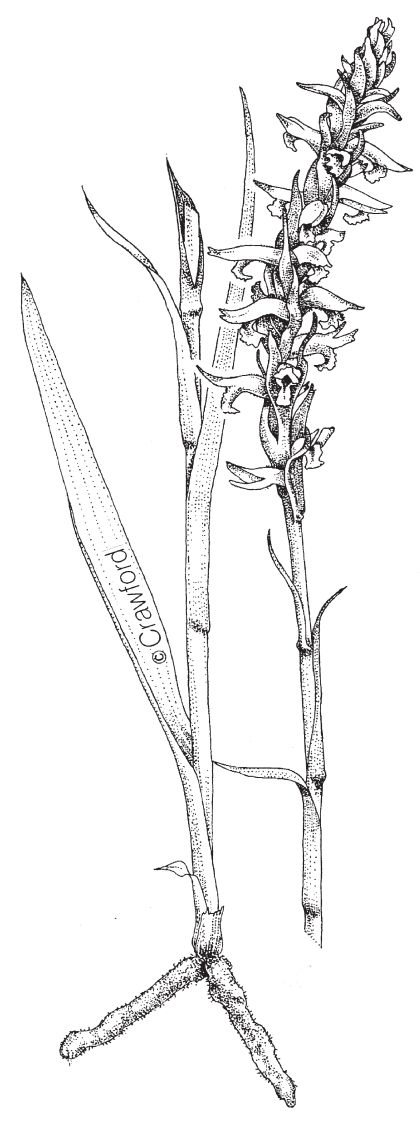

Ute Ladies' Tresses

(Spiranthes diluvialis)

Illustration by Carolyn Crawford our website.

\section{Table of Contents}

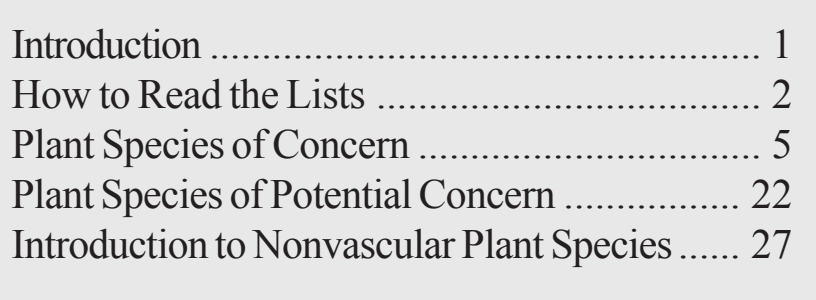

Bryophytes .................................................... 28

Lichens ……................................................ 30

Map of Montana Counties ................................ 33

Forest Service Sensitive Plant List ..................... 34

BLM Sensitive Plant List .................................. 34 
Some changes will likely be made to state and global ranks as new information comes in, and changes made between publications may be reflected in data queries to the MTNHP website: http://nhp.nris.state.mt.us/.

Our on-line Rare Plant Field Guide provides information on species' characteristics, identification, habitat, distribution, and references, as well as technical illustrations and photographs of the plants and their habitats. We are currently in the process of adding expanded habitat information, and sections on ecology and management, as well as a brief justification for the state rank. The Rare Plant Field Guide primarily covers Species of Concern, however some Potential Concern species are also included.

Distributional data on Plant Species of Concern can be queried on-line by county, topographic quadrangle (7.5') and public land management unit. A link to the NatureServe website provides access to information on the status of species throughout North America, assembled from state and provincial Natural Heritage databases (http:/ /www.natureserve.org/). More detailed information or additional assistance can be requested from MTNHP using the Information Request function on our website, or by phone, e-mail or mail.

\section{Additions to the Species of Concern List}

Senecio spribillei

\section{Deletions from the Species of Concern List}

(Downgraded to Species of Potential Concern)

Allotropa virgata

Botrychium montanum

Carex livida

Chrysosplenium tetrandrum

Lycopodium sitchense

Castilleja gracillima

Oenothera pallida var. idahoensis

\section{How To Read The Lists}

Species that have been added to the Concern or Potential Concern lists are bolded and underlined. Where only the state rank has been changed, that rank is bolded. Changes in global ranks are not highlighted. Also provided are federal designations under the U.S. Endangered Species Act and administrative designations assigned by the U.S. Forest Service or Bureau of Land Management; any changes to these status designations since our last publication are not highlighted.

The lists are organized alphabetically by scientific name. Common names are also provided for all vascular plants, and family names are included for Species of Concern. We have included a number of synonyms recognized as the most current nomenclature or as alternative taxonomic treatments by NatureServe (following Kartesz 1994 and Kartesz 1999) to facilitate searches using NatureServe Explorer (follow the link on our home page). Older names are retained, since they are still used in regional floras; newer names are given in parentheses. We have dropped variety and subspecies designations for many species that are significant in Montana at the species level, regardless of which infraspecific taxa occur (for many of these, only one variety or subspecies is known in Montana). This eliminates potential confusion about whether other subspecies/ varieties, if found, would be included in the status designation. It also minimizes identification to the level of variety and subspecies, which is often difficult with commonly available references. Information on infraspecific taxa will continue to be maintained in our databases.

\section{Heritage Program Ranks}

The international network of Natural Heritage Programs employs a standardized ranking system to denote global (range-wide) and state status (NatureServe 2002).

Species are assigned numeric ranks ranging from 1 (high risk) to 5 (demonstrably secure), reflecting the relative degree of risk, based upon available information.

A number of factors are considered in assigning ranks the number, size and distribution of known occurrences or populations, trends (if known), habitat sensitivity, and definable threats. Factors in a species' life history that make it especially vulnerable are also considered (e.g., dependence on a specific pollinator).

Montana Plant Species of Concern are those with a state ranking of S1 through S2 (including S2S3) or SH (known only from historical records). Species ranked S3 (including S3S4), SU, SR or other special rank designations are treated as Species of Potential Concern. Rank definitions are given below and reflect some updates in terminology in an attempt to avoid terms like "imperiled" that may be perceived as implying "endangered" or "threatened". The meaning and criteria for ranks remain unchanged, to maintain consistency with international standards.
Rank
G1 S1 
G2 S2

At risk because of very limited and potentially declining population numbers and/or habitat, making it vulnerable to global extinction or extirpation in the state.

G3 S3 Potentially at risk because of limited range, populations and/or habitat, even though it may be abundant in some areas.

G4 S4 Apparently secure, though it may be quite rare in parts of its range, and/or suspected to be declining.

G5 S5 Demonstrably secure, though it may be quite rare in parts of its range.

GU SU Possibly at risk, but status uncertain; more information is needed.

GH SH Historical, known only from records over 50 year ago; may be rediscovered.

GX SX Believed to be extinct globally or extirpated in the state; historical records only.

SR Reported within the state but the documentation has not been confirmed/verified.

SA Native in nearby states, but in Montana believed to be accidentally introduced, deliberately planted, or escaped from plantings.

HYB Recurrent hybrids.

\section{Combination Ranks}

G\#G\# or S\#S\#

Indicates a range of uncertainty about the status of the species.

\section{Sub-rank}

T\# Rank of a subspecies or variety; appended to the global rank of the full species, e.g. G4T3.

\section{Qualifiers}

Q Distinctiveness of the taxon is questionable; appended to the global rank, e.g. G3Q.

? Denotes uncertainty; inexactness.

\section{Federal Designations}

FISH AND WILDLIFE SERVICE

(U.S. Endangered Species Act)

This column indicates the status of a taxon under the federal Endangered Species Act of 1973 (16 U.S.C.A. § 1531-1543 (Supp. 1996)). Note: The Category 2 Candidates designation was dropped in 1996 by the USFWS, and former Candidate 2 species no longer have any formal status under ESA. Regulatory aspects of the Endangered Species Act affect plants only when they occur on federal lands or are affected by federal actions.

\section{LE Listed endangered}

LT Listed threatened

Howellia aquatilis

Silene spaldingii

Spiranthes diluvialis

P Proposed endangered or threatened

C Candidate with listing warranted but precluded

Botrychium lineare

\section{FOREST SERVICE}

The U.S. Forest Service Manual (2670.22) defines the status of sensitive species on Forest Service lands. The Regional Forester (Northern Region) designates sensitive species on National Forests in Montana; these designations were last updated in 1999, and a full list is included on page 34. They apply only on USFS-administered lands.

S Sensitive species, subspecies or varieties, for which the Regional Forester has determined there is a concern for population viability range-wide or in the region.

\section{BUREAU OF LAND MANAGEMENT}

The BLM 6840 Manual defines the status of species on Bureau of Land Management lands. The Montana State Office of the BLM assigned these designations in 1996, and a full list is included on page 34. They apply only on BLM-administered lands.

S Sensitive species are proven imperiled in at least part of their ranges and are documented to occur on BLM lands.

W Watch species are either known to be imperiled and suspected to occur on BLM lands, suspected to be imperiled and documented on BLM lands, or needing further study for other reasons. 


\section{County Distribution}

County listings have been dropped from the Species of Concern List to make formatting easier to read, and to reduce size. A listing that includes county distributions for Species of Concern is still provided and can be downloaded and printed from the MTNHP website at http://

nhp.nris.state.mt.us/. County distributions can also be queried for any Species of Concern, and queries to the databases will reflect new information as it is added to the system throughout the year.

\section{Range Notes}

Because state- and range-wide distribution patterns are important in assessing and understanding species' status, we have categorized distribution patterns in "range notes" for Species of Concern.

SE State Endemic - known to occur only in Montana.

RE Regional Endemic - restricted to the region, occurring in Montana and just one or very few other states or provinces.

D Disjunct - occurs in Montana well outside the principle or normal range of the species, separated by distances that preclude gene flow.

P Peripheral - occurs in Montana at the outer margins of its contiguous range.

S Sparse - Montana is part of a larger range in which the species is sparsely or unevenly distributed.

\section{Acknowledgements}

We would like to gratefully acknowledge the many people who contributed information on plant species' occurrences and distribution throughout Montana over the years - those contributions are the building blocks of the MTNHP databases and this publication. Special thanks go to the botanists who reviewed and provided comments on current changes to listings and status ranks, including Peter Lesica, Maria Mantas, John Pierce, Steve Shelly and Jennifer Whipple.

\section{Selected References}

Dorn, R.D. 1984. Vascular Plants of Montana. Mountain West Publishing, Cheyenne, WY. 276 pp.
Flora of North America Editorial Committee, eds. 1993+. Flora of North America North of Mexico. 4+ vols. New York and Oxford.

Heidel, B. 1999. Montana plant species of special concern. Montana Natural Heritage Program, Helena. 26pp.

Heidel, B. 2001. Montana plant species of concern. Montana Natural Heritage Program, Helena. 38pp.

Kartesz J.T. 1994. A synonymized checklist of the vascular flora of the United States, Canada, and Greenland. 2nd ed. 2 vols. Portland, (OR): Timber Press.

Kartesz, J.T. 1999. A Synonymized Checklist and Atlas with Biological Attributes for the Vascular Flora of the United States, Canada, and Greenland. First Edition. In: Kartesz, J.T., and C.A. Meacham. Synthesis of the North American Flora, Version 1.0. North Carolina Botanical Garden, Chapel Hill, NC.

Lesica, P., G. Moore, K.M. Peterson, and J.H. Rumely. 1984. Vascular plants of limited distribution in Montana. Montana Academy of Science Monograph No. 2.

Lesica, P. and J.S. Shelly. 1991. Sensitive, threatened and endangered vascular plants of Montana. Montana Natural Heritage Program, Montana State Library, Helena, Montana.

NatureServe Explorer: An on-line encyclopedia of life [web application]. 2002. Version 1.6 . Arlington, Virginia, USA: NatureServe. Available: http:// www.natureserve.org/explorer. (Accessed: April $15,2003)$.

The preferred citation for this document is:

Montana Natural Heritage Program. 2003. Plant Species of Concern. MTNHP, Helena. 34 pp.

The Montana Natural Heritage Program

P.O. Box 201800

1515 East Sixth Avenue

Helena, MT 59620-1800

(406) 444-5354 Fax (406) 444-0581

http://nhp.nris.state.mt.us/

Email Address: mtnhp@state.mt.us 


\section{Vascular Plant Species of Concern}

\section{Scientific Name}

Common Name
Sweetflag

Adoxa moschatellina

Musk-root

Agastache cusickii

Cusick's Horse-mint

Allium acuminatum

Tapertip Onion

Allium columbianum

Columbia Onion

Allium parvum

Small Onion

Allium simillimum

Dwarf Onion

Alnus rubra

Red Alder

Amerorchis rotundifolia

Round-leaved Orchis

Ammannia coccinea

Scarlet Ammannia

Amorpha canescens

Lead Plant

Antennaria densifolia

Dense-leaved Antennaria

Aquilegia brevistyla

Short-styled Columbine

Aquilegia formosa

Sitka Columbine

Arabis demissa

Daggett Rockcress

Arabis fecunda

Sapphire Rockcress

Arabis lyrata

Lyre-leaf Rockcress

Arctostaphylos patula

Green-leaf Manzanita

Asclepias incarnata

Swamp Milkweed

Asclepias ovalifolia

Ovalleaf Milkweed

Asclepias stenophylla

Narrowleaf Milkweed

Asplenium trichomanes

Maidenhair Spleenwort
Family

Acoraceae G5

Global
Rank

Adoxaceae G5

Lamiaceae

G2G3

Liliaceae

G5

Liliaceae

G3

Liliaceae

G5

Liliaceae

G4

Betulaceae

G5

Orchidaceae

G5

Lythraceae

G5

Fabaceae

G5

Asteraceae G3

Ranunculaceae

G5

Ranunculaceae

G5

Brassicaceae

G5

Brassicaceae

G2

Brassicaceae

G5

Ericaceae

G4

Asclepiadaceae G5

Asclepiadaceae G5?

Asclepiadaceae G4G5

Aspleniaceae

G5
State FWS FS

Rank Status Status

S 1

S2

S 1

S 1

S1

S2

S 1

S 1

S2S3

SH

$\mathrm{SH}$

S 1

S2

S 1

S 1

S2

$\mathrm{SH}$

S 1

S 1

S 1

S1

S

W

P

S

P

D

D

P

W P

S S SE

P

D

P

$\begin{array}{llll} & \text { S } & \text { W } & \text { P }\end{array}$

W P 


\section{Vascular Plant Species of Concern}

\section{Scientific Name}

Common Name

$\begin{array}{ll}\text { Family } & \begin{array}{l}\text { Global } \\ \text { Rank }\end{array}\end{array}$

Alkali Aster

Astragalus aretioides

Sweetwater Milkvetch

Astragalus barrii

Barr's Milkvetch

Astragalus ceramicus var. apus

Painted Milkvetch

Astragalus convallarius

Lesser Rushy Milkvetch

Astragalus geyeri var. geyeri

Geyer's Milkvetch

Astragalus grayi

Gray's Milkvetch

Astragalus lackschewitzii

Lackschewitz' Milkvetch

Astragalus oreganus

Wind River Milkvetch

Astragalus racemosus

Raceme Milkvetch

Astragalus scaphoides

Bitterroot Milkvetch

Astragalus terminalis

Railhead Milkvetch

Athysanus pusillus

Sandweed

Atriplex truncata

Wedge-leaved Saltbush

Bacopa rotundifolia

Roundleaf Water-hyssop

Balsamorhiza hookeri

Hooker's Balsam-root

Balsamorhiza macrophylla

Large-leafed Balsam-root

Bidens beckii (Megalodonta beckii var. beckii)

Beck Water-marigold

Boisduvalia densiflora

Dense Spike-primrose

Botrychium ascendens

Upward-lobed Moonwort

Botrychium campestre

Prairie Dunewort

Botrychium crenulatum

Wavy Moonwort
Asteraceae

G4

Fabaceae

G4

Fabaceae

G3

Fabaceae

G4T3

Fabaceae

G5

Fabaceae

G4?T4?

Fabaceae

G4?

Fabaceae

G2

Fabaceae

G4?

Fabaceae

G5

Fabaceae

G3

Fabaceae

G3

Brassicaceae

G4

Chenopodiaceae G5

Scrophulariaceae G5

Asteraceae G5

Asteraceae G3G5

G4G5T4

Asteraceae

G4G5T4

Onagraceae

G5

Ophioglossaceae G2G3

Ophioglossaceae G3G4

Ophioglossaceae G3

$\begin{array}{lll}\text { State } & \text { FWS } & \text { FS } \\ \text { Rank } & \text { Status } & \text { Status }\end{array}$

SS

BLM Range Status Notes

S 1

$\mathrm{P}$

S2

W

RE

S 2S 3

S

W

RE

S 1

S RE

S2

W P

S2

S

D

RE

RE

S2

S

W RE

S2

P

S2

S

S

RE

S2

S RE

S 1

S

P

S1

W S

S 1

W S

$\mathrm{S} 1$

P

S 1

S 2

P

P

S 1

S

S

S 1

S

S2

S

S 


\section{Vascular Plant Species of Concern}

\section{Scientific Name}

Common Name

Botrychium hesperium

Western Moonwort

Botrychium lineare

Linearleaf Moonwort

Botrychium pallidum

Pale Moonwort

Botrychium paradoxum

Peculiar Moonwort

Botrychium pedunculosum

Stalked Moonwort

Botrychium spathulatum

Spoon-leaf Moonwort

Brasenia schreberi

Watershield

Braya humilis

Low Braya

Calochortus bruneaunis

Bruneau Mariposa Lily

Camissonia andina

Obscure Evening -primrose

Camissonia parvula

Small Camissonia

Cardamine oligosperma var. kamtschatica

Few-seeded Bittercress

Carex amplifolia

Big-leaf Sedge

Carex chalciolepis

(C. heteroneura var. chalciolepis)

Copper-scale Sedge

Carex chordorrhiza

Creeping Sedge

Carex comosa

Bristly Sedge

Carex crawei

Craw's Sedge

Carex gravida

Pregnant Sedge

Carex idahoa

Idaho Sedge

Carex incurviformis

Maritime Sedge

Carex lacustris

Lake-bank Sedge

$\begin{array}{ll}\text { Family } & \begin{array}{l}\text { Global } \\ \text { Rank }\end{array}\end{array}$

Ophioglossaceae G3G4

Ophioglossaceae G1

Ophioglossaceae G3

Ophioglossaceae G2

Ophioglossaceae G2G3

Ophioglossaceae G3

Cabombaceae G5

Brassicaceae

G5

Liliaceae

G5

Onagraceae

G4

Onagraceae

G5

Brassicaceae

G5T3T5

Cyperaceae

G4

Cyperaceae

G5T4T5

Cyperaceae

G5

Cyperaceae

G5

Cyperaceae

G5

Cyperaceae

G5

Cyperaceae

G2Q

Cyperaceae

G4G5

Cyperaceae

G5

S1

S2

S 1

S 1

S2

S 1

S 1

S2

S 1

S2

S1

S1

S2

S2

$\mathrm{S} 1$

S2

S 1
State FWS FS BLM Range

Rank Status Status Status Notes

$\mathrm{S}$

$\mathrm{S}$

PT

S

S

S

S

S

S

S

S

S

S

SH

P

$\mathrm{P}$

P

S

$\mathrm{P}$

$\mathrm{P}$

S

$\mathrm{P}$

S

$\mathrm{S} \quad \mathrm{S}$

W P

2

S

D 


\section{Vascular Plant Species of Concern}

\section{Scientific Name}

Common Name

Global
Rank

Cyperaceae

Cyperaceae

Cyperaceae

Cyperaceae

Cyperaceae

Cyperaceae

G5

G5T?

G4

G5T3Q

G5

G5T?Q

Cyperaceae G4

Cyperaceae

G5?

Cyperaceae

G5

G5

Cyperaceae

Cyperaceae

G2

Cyperaceae

G4

Cyperaceae

G5

Cyperaceae

G4G5

Cyperaceae

G5

Scrophulariaceae G4

Scrophulariaceae G3G4

Scrophulariaceae G5

Rhamnaceae

G5

Celastraceae

G5

Gentianaceae

G5
S 1

S 1

$\mathrm{SH}$

S 1

State FWS FS

Rank Status Status

S2

S 1

W

D

$\mathrm{P}$

W P

D

$\mathrm{P}$

P

P

S

S

S

P

$\mathrm{P}$

P

RE

S2

S

RE

S2

$\mathrm{P}$

SH

W P

S 1

W P

Bittersweet

Centaurium exaltatum

Western Centuary 


\section{Vascular Plant Species of Concern}

Scientific Name

Common Name
Family

Primulaceae

Rosaceae

G5T3T5

Chenopodiaceae G3G4

Asteraceae

G5T1

Asteraceae

G4

Asteraceae

G2Q

Onagraceae

G5

Portulacaceae

G4

Capparaceae

G5

Polemoniaceae G5

Fumariaceae

Boraginaceae

G4

Boraginaceae

G4?

Boraginaceae

G4?

Cyperaceae

G5

Cyperaceae

G5

Cyperaceae

G5

Cyperaceae

G5

Orchidaceae

G4

Orchidaceae

G5

Orchidaceae

G4G5

Dryopteridaceae G5

$\begin{array}{lll}\text { State } & \text { FWS } & \text { FS } \\ \text { Rank } & \text { Status } & \text { Status }\end{array}$

S2

S1S2

W P

S 1

W S

S 1

S2

S2

S

SE

S2

S

P

S 1

S

P

S 1

W P

S 1

S1

S2

W

S

$\mathrm{SH}$

W

S

W S

S 1

$\mathrm{SH}$

S

S

S1

S2

W

P

S2

S

P

S 2S3

S

W

S

S2

S

P

Sparrow's-egg Lady's Slipper

Cystopteris montana

$\mathrm{SH}$

Mountain Bladder Fern 


\section{Vascular Plant Species of Concern}

\section{Scientific Name}

Common Name

Family

Fabaceae

Nine-anther Dalea

Dalea villosa

Silky Prairie Clover

Dichanthelium oligosanthes var. scribnerianum Scribner's Panic Grass

Downingia laeta

Great Basin Downingia

Draba densifolia

Dense-leaf Draba

Draba fladnizensis

White Arctic Draba

Draba globosa

Round-fruited Draba

Draba macounii

Macoun's Draba

Draba porsildii

Porslid's Draba

Draba ventosa

Wind River Draba

Drosera anglica

English Sundew

Drosera linearis

Linear-leaved Sundew

Dryas integrifolia

Entire-leaved Avens

Dryopteris cristata

Buckler Fern

Eleocharis rostellata

Beaked Spikerush

Elodea longivaginata

Long Sheath Waterweed

Elymus flavescens (Leymus flavescens)

Sand Wildrye

Elymus innovatus (Leymus innovatus)

Northern Wildrye

Epipactis gigantea

Giant Helleborine

Erigeron asperugineus

Idaho Fleabane

Erigeron eatonii

Eaton's Daisy

Erigeron evermannii

Everman Fleabane
Fabaceae

Poaceae

Campanulaceae G5

Brassicaceae G5

Brassicaceae

G4

Brassicaceae

G3

Brassicaceae

G3G4

Brassicaceae

G3G4Q

Brassicaceae

G3

Droseraceae

G5

Droseraceae

G4

Rosaceae

G5

Dryopteridaceae G5

Cyperaceae G5

Hydrocharitaceae G4G5

Poaceae

G4

Poaceae

G5

Orchidaceae

G3G4

Asteraceae

G4

Asteraceae

G5

Asteraceae

G4
S 1

S

BLM Range

Rank Status Status Status Notes

S 1

W

$P$

S 1

W

$\mathrm{P}$

S 1

W

S

S 1

W S

S2

S

S

W RE

$\mathrm{P}$

D

S

S

$\mathrm{P}$

$\mathrm{P}$

P

S2

S2

W

S

S 1

S

P

S 1

S

P

S2

S 1

S 1

P

RE

(c) 2003 Montana Natural Heritage Program 


\section{Vascular Plant Species of Concern}

\section{Scientific Name}

Common Name

Erigeron formosissimus

Beautiful Fleabane

Erigeron lackschewitzii

Lackschewitz' Fleabane

Erigeron leiomerus

Smooth Fleabane

Erigeron linearis

Linear-leaf Fleabane

Erigeron tener

Slender Fleabane

Eriogonum caespitosum

Mat Buckwheat

Eriogonum salsuginosum

Smooth Buckwheat

Eriogonum visheri

Visher's Buckwheat

Eriophorum callitrix

Sheated Cotton-grass

Eriophorum gracile

Slender Cotton-grass

Eupatorium maculatum

Joe-pye Weed

Eupatorium occidentale (Ageratina occidentalis)

Western Boneset

Euphrasia arctica var. disjuncta (E. disjuncta)

Disjunct Eyebright

Eustoma grandiflorum (E. russellianum)

Showy Prairie-gentian

Festuca vivipara

Viviparous Fescue

Gentiana glauca

Glaucous Gentian

Gentianopsis macounii

Macoun's Gentian

Gentianopsis simplex

Hiker's Gentian

Githopsis specularioides

Common Blue-cup

Glossopetalon nevadense

(Glossopetalon spinescens var. aridum)

Spiny Greenbush

Goodyera repens

Northern Rattlesnake-plantain
Family

Asteraceae

Global

Rank

Asteraceae

G3

Asteraceae

G4

Asteraceae

G5

Asteraceae

G4

Polygonaceae G5

Polygonaceae

G4?

Polygonaceae

G3

Cyperaceae

G5

Cyperaceae

G5

Asteraceae

G5

Asteraceae G4

Scrophulariaceae G5

Gentianaceae

G5

Poaceae

G4G5

Gentianaceae

G4G5

Gentianaceae G5

Gentianaceae

G4

Campanulaceae G5

CrossosomataceaeG 5 T 5 ?

Orchidaceae

G5

S 2S 3

S

S
RE

$\mathrm{P}$

P

P

P

S P

RE

S

$\mathrm{P}$

W P

W D

P

P

$\begin{array}{llll}\text { S } 1 & \text { S } & \text { W } & \text { P }\end{array}$

$\begin{array}{llll}\text { S } 1 & \text { S } & \text { W } & \text { P }\end{array}$

S $12 \mathrm{P}$

S 1

S

P 


\section{Vascular Plant Species of Concern}

Scientific Name

Common Name
Global

Rank
Scrophulariaceae G4

Bractless Hedge-hyssop

Grayia spinosa

Spiny Hopsage

Grindelia howellii

Howell's Gum-weed

Gymnosteris parvula

Small-flower Gymnosteris

Halimolobos perplexa

Puzzling Rockcress

Haplopappus aberrans (Tonestus aberrans)

Idaho Goldenweed

Haplopappus carthamoides var. subsquarrosus

(Pyrrocoma carthamoides var. subsquarrosa)

Beartooth Large-flowered Goldenweed

Haplopappus macronema var. macronema

(Ericameria discoidea var. discoidea)

Discoid Goldenweed

Haplopappus nanus (Ericameria nana)

Dwarf Goldenweed

Haplopappus pygmaeus (Tonestus pygmaeus)

Pygmy Goldenweed

Hemicarpha drummondii (Lipocarpha drummondii)

Drummond's Hemicarpha

Heteranthera dubia

Water Star-grass

Heterocodon rariflorum

Western Pearl-flower

Howellia aquatilis

Water Howellia

Hutchinsia procumbens

Hutchinsia

Idahoa scapigera

Scalepod

Ipomopsis congesta ssp. crebrifolia

Ballhead Gilia

Ipomopsis minutiflora

Small-flower Standing-cypress

Juncus acuminatus

Tapered Rush

Juncus albescens

Three-flowered Rush

Juncus covillei var. covillei

Coville's Rush
Chenopodiaceae G5

Asteraceae

G3

Polemoniaceae

G4

Brassicaceae

G4

Asteraceae

G3

Asteraceae

G4G5T2T3

Asteraceae

G4G5T4

Asteraceae G4

G4G5

Campanulaceae G5

Campanulaceae

G2

Brassicaceae

G5

Brassicaceae

G5

Polemoniaceae

G5T3T4

Polemoniaceae

G2G3

Juncaceae

G5

Juncaceae

G5

Juncaceae

G4G5T5
S1

S 1

SH

$\mathrm{SH}$

S 1

S 1

RE

$\mathrm{P}$

SE

RE

RE

P

$\mathrm{P}$

$\mathrm{P}$

$\mathrm{P}$

S

S

$\begin{array}{llll}\text { S2 } & \text { LT } & \text { S } & \text { S }\end{array}$

$\begin{array}{lll}\text { S1 } & \text { W } & \text { P }\end{array}$

S1 S

P

RE

D

$\mathrm{S}$

S

P 


\section{Vascular Plant Species of Concern}

\section{Scientific Name}

Common Name

Family

Juncaceae

Coville's Blunt Rush

Juncus hallii

Hall's Rush

Kalmia polifolia

Pale laurel

Kelloggia galioides

Kelloggia

Kobresia macrocarpa (K. sibircia)

Large-fruited Kobresia

Kobresia simpliciuscula

Simple Kobresia

Kochia americana

Red Sage

Koenigia islandica

Island Koenigia

Lagophylla ramosissima

Slender Hareleaf

Lathyrus bijugatus

Latah Tule Pea

Leptodactylon caespitosum

Leptodactylon

Lesquerella carinata var. languida

Garnet Bladderpod

Lesquerella douglasii

Douglas Lesquerella

Lesquerella humilis

Bitterroot Bladderpod

Lesquerella lesicii

Lesica's Bladderpod

Lesquerella paysonii

Payson Bladderpod

Lesquerella pulchella

Beautiful Bladderpod

Lewisia columbiana

Columbia Lewisia

Lewisia pygmaea var. nevadensis (L. nevadensis)

Nevada Lewisia

Lilaea scilloides

Flowering Quillwort

Liparis loeselii

Loesel's Twayblade

Lobelia spicata

Pale-spiked Lobelia

Juncaceae

Ericaceae

Rubiaceae

Fabaceae

$\begin{array}{llllll}\text { Global } & \text { State } & \text { FWS } & \text { FS } & \text { BLM } & \text { Range } \\ \text { Rank } & \text { Rank } & \text { Status } & \text { Status } & \text { Status } & \text { Notes }\end{array}$

G4G5T4

S1

$\mathrm{P}$

G4G5

S2

S

P

G5

S1

S

P

G5

S 1

P

Cyperaceae G5

S 1

P

Cyperaceae G5

S2

S

Chenopodiaceae G5

S 1

P

Polygonaceae G4

S 1

D

Asteraceae G5

S 1

P

G4

S1

$\mathrm{S}$

RE

Polemoniaceae G4

S2

W

$\mathrm{P}$

Brassicaceae

G3G4T1

S1

S

SE

Brassicaceae

G4?

S 1

P

Brassicaceae G1

S1

S

SE

Brassicaceae

G1

S1

S

SE

Brassicaceae G3

S 1

S

RE

Brassicaceae

G2

S2

S

S

SE

Portulacaceae

G4

S 1

RE

Portulacaceae

G4

S1

P

Juncaginaceae G5?

S1

P

Orchidaceae G5

S 1

S

P

Campanulaceae G5

S 1

$P$

P

P

P

S

P

D

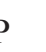

P




\section{Vascular Plant Species of Concern}

\section{Scientific Name}

Common Name

Lomatium attenuatum

Taper-tip Desert-parsley

Lomatium geyeri

Geyer's Biscuitroot

Lomatium nuttallii

Nuttall's Desert-parsley

Lomatogonium rotatum

Felwort

Lycopodium dendroideum

Treelike Clubmoss

Lycopodium inundatum

Northern Bog Clubmoss

Lycopodium lagopus

Running Pine

Maianthemum canadense

Wild Lily-of-the-valley

Malacothrix torreyi

Desert Dandelion

Mentzelia montana

White-bract Stickleaf

Mentzelia nuda

Bractless Mentzelia

Mentzelia pumila

Dwarf Mentzelia

Mertensia bella

Oregon Bluebells

Mimulus breviflorus

Short-flowered Monkeyflower

Mimulus nanus

Dwarf Purple Monkeyflower

Mimulus patulus

Washington Monkeyflower

Mimulus primuloides

Primrose Monkeyflower

Mimulus ringens

Square-stem Monkeyflower

Najas guadalupensis

Guadalupe Water-nymph

Nama densum

Nama

Nuttallanthus texanus

Blue Toadflax

Nymphaea tetragona ssp. leibergii (N. leibergii)

Pygmy Water-lily

Family

Apiaceae

Apiaceae

Apiaceae

G3

Gentianaceae

G5

Lycopodiaceae G5

Lycopodiaceae G5

Lycopodiaceae G5

Liliaceae

G5

Asteraceae

G4

Loasaceae

G4

Loasaceae

G5

Loasaceae

G4

Boraginaceae

G4

Scrophulariaceae G4

Scrophulariaceae G5

Scrophulariaceae G2Q

Scrophulariaceae G4

Scrophulariaceae G5

Najadaceae

G5

Hydrophyllaceae G5

Scrophulariaceae G4G5

Nymphaeaceae G5
State FWS FS BLM Range

Rank Status Status Status Notes

S2

$\mathrm{S}$

RE

S2

S

RE

$\mathrm{S} 1$

W P

S 1

S

W

S

S 1

S

$\mathrm{P}$

S 1

S

P

S 1

$\mathrm{P}$

SH

$\mathrm{P}$

S1

S

D

$\mathrm{SH}$

P

S1

W P

S2

W P

S 1

S

D

S 1

P

S 1

P

S1

S

D

S2

S

P

S 1

P

S1

W P

S 1

S P

S 1

W P

S 1

D 


\section{Vascular Plant Species of Concern}

\section{Scientific Name}

Common Name

$\begin{array}{ll}\text { Family } & \begin{array}{l}\text { Globa } \\ \text { Rank }\end{array}\end{array}$

Ophioglossaceae G5

Apiaceae

Fabaceae

G5T3

Fabaceae

G5T?

Fabaceae

G5

Fabaceae

G4

Papaveraceae

G5T3T4

Papaveraceae

G3

Scrophulariaceae G5

Scrophulariaceae G4T4

Scrophulariaceae G4

Scrophulariaceae G5?

Scrophulariaceae G3

Scrophulariaceae G4

Scrophulariaceae G5

Asteraceae G5

Hydrophyllaceae G3G4

Hydrophyllaceae G4

Hydrophyllaceae G3G4

Poaceae

G5

Polemoniaceae G4

Polemoniaceae G2
State FWS FS BLM Range

Rank Status Status Status Notes

$\mathrm{S}$

S2

S 1

S 1

S 1

S 1

S

S S

S 2S3 RE

$\mathrm{P}$

RE

S

P
S2

W P

S 1

RE

S2

$\mathrm{P}$

S 1

$\mathrm{P}$

S2

$\mathrm{S} 1$

S 1

$\mathrm{S} 1$

S2

$\mathrm{SH}$

S 1

S2

S2

W P

Plains Phlox

Phlox kelseyi var. missoulensis (P. missoulensis)

Missoula Phlox 


\section{Vascular Plant Species of Concern}

\section{Scientific Name}

Common Name

\begin{tabular}{ll} 
Family & $\begin{array}{l}\text { Globa } \\
\text { Rank }\end{array}$ \\
\hline
\end{tabular}

Brassicaceae

G5

Brassicaceae

G5T2

Boraginaceae

G4

G4

Poaceae

Poaceae

G5?T1

Polygonaceae

G5T4

Dryopteridaceae G4

Dryopteridaceae G5

PotamogetonaceaeG 5

Rosaceae

G4

Rosaceae

G4G5

Rosaceae

G4

Rosaceae

G5T4

Rosaceae

G5

Primulaceae

G2

Primulaceae

G4G5

Rosaceae

G5

Asteraceae

G4

Poaceae

G4

Fagaceae

G5

Ranunculaceae

G4G5

Ranunculaceae

G4

$\begin{array}{lll}\text { State } & \text { FWS } & \text { FS } \\ \text { Rank } & \text { Status } & \text { Status }\end{array}$

FS

BLM Range Status Notes

S2

RE

RE

S1

S 1

S 1

S 2

S

S

P

P

S2

S

P

RE

S

W S

S

$\mathrm{S}$

S $1 \quad$ W $\quad$ RE

S2

W P

S 1

P

S2

$\mathrm{S} 1$

$\mathrm{P}$

S 1

S P

W S

S2

$S$

Heart-leaved Buttercup

Ranunculus gelidus

Ranunculaceae

Arctic Buttercup

(c) 2003 Montana Natural Heritage Program 


\section{Vascular Plant Species of Concern}

\section{Scientific Name}

Common Name

Ranunculus hyperboreus

High-arctic Buttercup

Ranunculus jovis

Jove's Buttercup

Ranunculus orthorhynchus

Straightbeak Buttercup

Ranunculus pedatifidus

Northern Buttercup

Ranunculus verecundus

Timberline Buttercup

Ribes cognatum

Shinyleaf Gooseberry

Ribes laxiflorum

Trailing Black Currant

Ribes triste

Swamp Red Currant

Ribes velutinum

Desert Gooseberry

Rorippa calycina

Persistent-sepal Yellow-cress

Rotala ramosior

Toothcup

Sagina nivalis

Arctic Pearlwort

Salix barrattiana

Barratt's Willow

Salix cascadensis

Cascade's Willow

Salix serissima

Autumn Willow

Satureja douglasii

Yerba Buena

Saussurea densa

Dwarf Saw-wort

Saussurea weberi

Weber's Saw-wort

Saxifraga apetala

Tiny Swamp Saxifrage

Saxifraga hirculus

Yellow Marsh Saxifrage

Saxifraga tempestiva

Storm Saxifrage

Scheuchzeria palustris

Pod Grass

\begin{tabular}{|c|c|c|c|c|c|c|}
\hline Family & $\begin{array}{l}\text { Global } \\
\text { Rank }\end{array}$ & $\begin{array}{l}\text { State } \\
\text { Rank }\end{array}$ & $\begin{array}{l}\text { FWS } \\
\text { Status }\end{array}$ & $\begin{array}{l}\text { FS } \\
\text { Status }\end{array}$ & $\begin{array}{l}\text { BLM } \\
\text { Status }\end{array}$ & $\begin{array}{l}\text { Range } \\
\text { Notes }\end{array}$ \\
\hline Ranunculaceae & G5 & $\mathrm{S} 1$ & & & & $\mathrm{P}$ \\
\hline Ranunculaceae & G4 & $\mathrm{S} 2$ & & $\mathrm{~S}$ & & $\mathrm{RE}$ \\
\hline Ranunculaceae & G5 & $\mathrm{S} 1$ & & & & $\mathrm{P}$ \\
\hline Ranunculaceae & G5 & $\mathrm{S} 1$ & & & $\mathrm{~W}$ & $\mathrm{~S}$ \\
\hline Ranunculaceae & G5 & $\mathrm{S} 2$ & & & & $\mathrm{~S}$ \\
\hline Grossulariaceae & G4Q & $\mathrm{S} 1$ & & & & $\mathrm{RE}$ \\
\hline Grossulariaceae & G5 & $\mathrm{S} 1$ & & & & $\mathrm{D}$ \\
\hline Grossulariaceae & G5 & $\mathrm{S} 1$ & & & & $\mathrm{P}$ \\
\hline Grossulariaceae & G5 & $\mathrm{S} 1$ & & & & $\mathrm{P}$ \\
\hline Brassicaceae & G3 & $\mathrm{S} 1$ & & & W & $\mathrm{RE}$ \\
\hline Lythraceae & G5 & $\mathrm{S} 1$ & & & & $\mathrm{P}$ \\
\hline Caryophyllaceae & G5 & $\mathrm{S} 1$ & & & & $\mathrm{D}$ \\
\hline Salicaceae & G5 & $\mathrm{S} 1$ & & $\mathrm{~S}$ & & $\mathrm{~S}$ \\
\hline Salicaceae & G4G5 & $\mathrm{S} 1$ & & & & $\mathrm{~S}$ \\
\hline Salicaceae & G4 & $\mathrm{S} 2$ & & & W & $\mathrm{P}$ \\
\hline Lamiaceae & G4 & $\mathrm{S} 2$ & & & & $\mathrm{P}$ \\
\hline Asteraceae & G3G4 & $\mathrm{S} 2$ & & & & $\mathrm{RE}$ \\
\hline Asteraceae & G2G3 & $\mathrm{S} 1$ & & $\mathrm{~S}$ & & $\mathrm{D}$ \\
\hline Saxifragaceae & G3Q & $\mathrm{S} 2$ & & & & $\mathrm{RE}$ \\
\hline Saxifragaceae & G5 & $\mathrm{S} 1$ & & & & $\mathrm{~S}$ \\
\hline Saxifragaceae & $\mathrm{G} 2$ & $\mathrm{~S} 2$ & & $\mathrm{~S}$ & & SE \\
\hline Scheuchzeriaceae & G5 & $\mathrm{S} 2$ & & $\mathrm{~S}$ & & $\mathrm{P}$ \\
\hline
\end{tabular}




\section{Vascular Plant Species of Concern}

Scientific Name

Common Name

Scirpus cespitosus

Tufted Club-rush

Scirpus heterochaetus

Slender Bulrush

Scirpus hudsonianus (Eriophorum alpinum)

Hudson's Bay Bulrush

Scirpus pumilus ssp. rollandii (S. rollandii)

Small clubrush

Scirpus subterminalis

Water Bulrush

Selaginella selaginoides

Low Spike-moss

Senecio amplectens

Clasping Groundsel

Senecio pauciflorus

Few-flowered Butterweed

$\underline{\text { Senecio spribillei }}$

Spribillei's Groundsel

Shoshonea pulvinata

Shoshonea

Sidalcea oregana

Oregon Checker-mallow

Silene spaldingii

Spalding's Campion

Sisyrinchium septentrionale

Northern Blue-eyed Grass

Solidago ptarmicoides

Prairie Aster

Solidago sparsiflora

Few-flowered Goldenrod

Sphaeralcea munroana

White-stemmed Globe-mallow

Sphaeromeria argentea

Chicken Sage

Sphenopholis intermedia

Slender Wedgegrass

Spiranthes diluvialis

Ute Ladies' Tresses

Sporobolus asper (S. compositus var. compositus)

Longleaf Dropseed

Sporobolus neglectus

Small Dropseed

Stellaria crassifolia

Fleshy Stitchwort

\begin{tabular}{lllllll} 
Family & $\begin{array}{l}\text { Global } \\
\text { Rank }\end{array}$ & $\begin{array}{l}\text { State } \\
\text { Rank }\end{array}$ & $\begin{array}{l}\text { FWS } \\
\text { Status }\end{array}$ & $\begin{array}{l}\text { FS } \\
\text { Status }\end{array}$ & $\begin{array}{l}\text { BLM } \\
\text { Status }\end{array}$ & $\begin{array}{l}\text { Range } \\
\text { Notes }\end{array}$ \\
\hline Cyperaceae & G5 & S2 & & S & & S
\end{tabular}

Cyperaceae G

Cyperaceae G5

S 1

P

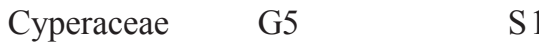

$\mathrm{P}$

Cyperaceae G4G5

2

S2

S

Selaginellaceae G

Asteraceae G4

S 1

$\mathrm{P}$

Asteraceae

G4G5

S 1

$\mathrm{P}$

Asteraceae $\quad \mathrm{G}$

$\underline{\text { S } 1}$

SE

Apiaceae G2G3

$\mathrm{S} 1$

$\mathrm{S} \quad \mathrm{S} \quad \mathrm{RE}$

Malvaceae G5

S 1

$\mathrm{P}$

Caryophyllaceae G2

S 1

LT

RE

Iridaceae

G3G4

S 1

$\mathrm{P}$

Asteraceae

G5

S 1

$\mathrm{P}$

Asteraceae

G5?

S 1

W

S

Malvaceae $\quad G$

S 1

P

Asteraceae G3G4

S2

S

$\mathrm{P}$

Poaceae

G5

S 1

W

P

Orchidaceae G2

S2

LT

W S

Poaceae

G5T5

SH

W P

Poaceae

G5

S 1

W P

Caryophyllaceae G5

S 1

W

S

S

S

$P$

$P$

RE

$P$

$P$

$P$

(c) 2003 Montana Natural Heritage Program 


\section{Vascular Plant Species of Concern}

\section{Scientific Name}

Common Name
Townsendia florifer

Showy Townsendia

Trifolium cyathiferum

Cup Clover

Trifolium eriocephalum

Woolly-head Clover

Trifolium gymnocarpon

Hollyleaf Clover

Utricularia intermedia

Flat-leaved Bladderwort

Vaccinium myrtilloides

Velvetleaf Blueberry

Veratrum californicum

California False-hellebore

Viburnum lentago

Nannyberry

Viguiera multiflora (Heliomeris multiflora)

Many-flowered Viguiera
Family

Global
Rank

Caryophyllaceae G5

Asteraceae G4

Poaceae

G5

Chenopodiaceae G5

Saxifragaceae G3

Asteraceae G4

Ranunculaceae G5

Brassicaceae

G2

Brassicaceae G4

Thelypteridaceae G5

Brassicaceae

G3

Liliaceae

G5

Asteraceae

G4

Asteraceae

G5

$\begin{array}{lllll}\text { State } & \text { FWS } & \text { FS } & \text { BLM } & \text { Range } \\ \text { Rank } & \text { Status } & \text { Status } & \text { Status } & \text { Notes }\end{array}$

S 1

W

S 1

W P

S 1

S 1

S2

W RE

S2

S S

S2

S

S

SH

S RE

S2

S2

S

S2

S

S2

S 1S 2

W

Fabaceae

G4

S 1

W

Fabacea

G5

S1

Fabaceae $\quad$ G4

Lentibulariaceae G5

S1

S 1

Ericaceae

G5

G5

S1

S

W

Caprifoliaceae G5

S 1

Asteraceae G4G5

S 1

$\mathrm{P}$

P

P

S

P

$\mathrm{P}$

$\mathrm{RE}$

$\mathrm{P}$

RE

P

P

P

$\mathrm{P}$

P

P

P

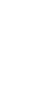

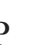

RE

S

S

RE

P

$\mathrm{P}$

$\mathrm{P}$

P

$\mathrm{P}$ 


\section{Vascular Plant Species of Concern}

\section{Scientific Name}

Common Name

Family

Violaceae

Rosaceae

Lemnaceae

G5

Apiaceae

\section{Global Rank}

G5?

G3

G5
State FWS FS BLM Range

Rank Status Status Status Notes

S1

$\mathrm{S} 1$

S2

W

S

Columbia Water-meal

Golden Alexanders

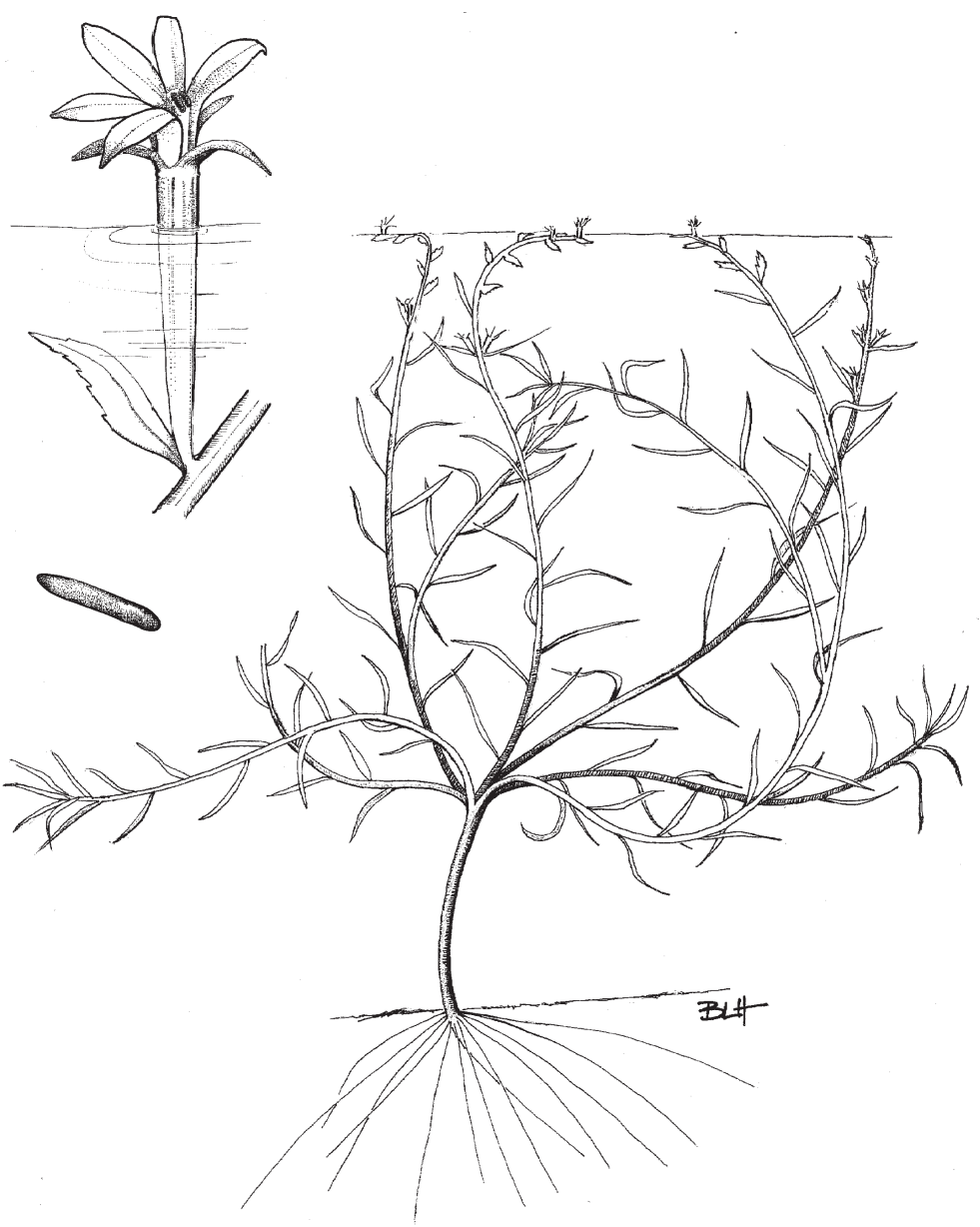

Water Howellia

(Howellia aquatilis)

Illustration by Bonnie Heidel 


\section{Plant Species of Potential Concern}

\section{April 2003}

Species of Potential Concern include several categories of species (and subspecific taxa). Some, while very limited in abundance or distribution in Montana, are not currently thought to be sufficiently rare or at risk to be Species of Concern; these have been ranked S3 or S3S4. Many are noteworthy because they are of limited global distribution and/or regional endemics (G3 or G3G4). A number of other plants of Potential Concern are of undetermined status and still under review (ranked SU), or have been reported to occur in Montana but need further verification (SR). A few species, while very rare in Montana, appear to be adventive - rare in the state but not considered native to the Montana flora (ranked SA). The remaining species on the Potential Concern list are a small number of recurrent hybrids (HYB).

Previous versions of this report included columns indicating categories or reasons for uncertainty. The format of this edition has been modified to better use state ranks for representing the different categories of Potential Concern plants, as referenced above. This condensed format has allowed us to add common names and agency status designations. The former categories correspond to ranks as described below:

\section{S3, S3S4 Limited abundance or distribution in} Montana, but not presently considered to be at risk (formerly Limited

\section{Distribution).}

SA Rare but considered likely to be adventive and not native to Montana (formerly Native?)

SR Reported to occur in Montana but needs further verification (formerly Verified? and In MT?).

SU Status undetermined or uncertain and still under review; this includes many plants for which data has not yet been fully assembled and reviewed (formerly Data Not Compiled), and others that may be quite rare or limited in distribution, but are not demonstrably vulnerable (formerly Vulnerable?).

HYB Recurrent hybrids.
Also previously included were a few species (denoted in the Publication column) that had not yet been named and formally described, as is required for scientific validity. Since, without valid names and references, there is no way of identifying or communicating about these plants, we have removed them from the list. We will continue to maintain records of their reported locations, and will add them if and when they are formally named and described (as recently occurred with Senecio spribillei).

In the past, the Heritage Program did not regularly keep track of occurrences for Species of Potential Concern. When a species was dropped from Special Concern status, occurrence data was removed from the active Heritage databases. We have now begun managing data on the distribution and status of Potential Concern plants; though it will take some time to reactivate old data records for "dropped" species and begin entering location data for others. The most immediate impact is that species downgraded from Species of Concern to Potential Concern will continue to be "tracked" in the Heritage data system and their occurrences reported through our data request process (with their Potential Concern status clearly indicated). We will also continue entering observational data that we receive for these species, so their status can be monitored in the future.

This publication identifies 220 Species of Potential Concern, including nine additions and five deletions. Of the five deletions, four are as yet unnamed Botrychium taxa and one is an unnamed Draba.

Further information on any Species of Potential Concern is available upon request. 


\begin{tabular}{|c|c|c|c|c|c|}
\hline Scientific Name & Common Name & $\begin{array}{c}\text { Global } \\
\text { Rank }\end{array}$ & $\begin{array}{l}\text { State } \\
\text { Rank }\end{array}$ & $\begin{array}{c}\text { FS } \\
\text { Status } \\
\end{array}$ & $\begin{array}{l}\text { BLM } \\
\text { Status }\end{array}$ \\
\hline Achillea sibirica & Siberian Yarrow & G5? & SA? & & \\
\hline Agastache foeniculum & Lavender Hyssop & G4G5 & SU & & \\
\hline Agoseris lackschewitzii & Pink Agoseris & G4 & S3 & & \\
\hline Agrimonia gryposepala & Tall Hairy Groovebur & G5 & SU & & \\
\hline Agrostis borealis (A. mertensii) & Northern Bentgrass & G5 & SU & & \\
\hline Allotropa virgata & Candystick & G4 & S3 & $\mathrm{S}$ & \\
\hline Amaranthus californicus & California Amaranth & G4 & SU & & \\
\hline Ambrosia acanthicarpa & Flat-spine Bursage & G5 & S3S4 & & $\mathrm{W}$ \\
\hline Ambrosia tomentosa & Skeleton-leaf Bursage & G4 & $\mathrm{SR}$ & & \\
\hline Amsinckia lycopsoides & Bugloss Fiddle-neck & G4? & SA? & & \\
\hline Antennaria aromatica & Aromatic Everlasting & G4 & S3S4 & & \\
\hline Antennaria neodioica ssp. neodioica & Northern Pussytoes & G4T5 & SU & & \\
\hline Antennaria pulcherrima & Showy Pussytoes & G4G5 & SU & & \\
\hline Arabidopsis salsuginea & Saltwater Cress & G4G5 & SR & & \\
\hline $\begin{array}{c}\text { Arabis pendulocarpa var. saximontana } \\
(\text { A. williamsii var. saximontana })\end{array}$ & Wind-river Rockcress & G5T5? & SR & & \\
\hline Arabis sparsiflora var. columbiana & Elegant Columbian Rockcress & G5T2T4 & SR & & \\
\hline Arabis sparsiflora var. subvillosa & Elegant Columbian Rockcress & G5T3T5 & SR & & \\
\hline $\begin{array}{l}\text { Artemisia longiloba } \\
\quad(\text { A. arbuscula ssp. longiloba })\end{array}$ & Alkali Sagebrush & G5T4 & SR & & \\
\hline Artemisia tilesii & Aleutian Mugwort & G5 & SR & & \\
\hline Asplenium trichomanes-ramosum & Green Spleenwort & G4 & SU & & \\
\hline Aster cusickii & Cusick Aster & G4? & $\mathrm{SR}$ & & \\
\hline Aster foliaceus var. lyallii & Lyall's Leafy-bracted Aster & G5T4? & SR & & \\
\hline Aster glaucodes & Gray Aster & G4G5 & $\mathrm{S} 3$ & & \\
\hline Aster simplex var. ramosissimus & Panicled Aster & G5T? & SR & & \\
\hline Astragalus chamaeleuce & Ground Milkvetch & G5 & S3 & & \\
\hline Astragalus kentrophyta var. kentrophyta & Spiny Milkvetch & G5T4 & S3 & & \\
\hline Astragalus lentiginosus & Sodaville Milkvetch & G5 & S3 & & \\
\hline Astragalus leptaleus & Park Milkvetch & G4 & S3 & & \\
\hline Astragalus miser var. crispatus & Timber Milkvetch & G5T3? & SU & & \\
\hline Astragalus platytropis & Broad-keeled Milkvetch & G5 & S3 & & \\
\hline Astragalus racemosus var. longisetus & Raceme Milkvetch & G5T4 & $\mathrm{SR}$ & & $\mathrm{W}$ \\
\hline Bidens comosa & Threelobed Beggarticks & G5 & SU & & $\mathrm{W}$ \\
\hline Bidens frondosa & Devil's Beggarticks & G5 & SU & & \\
\hline Bidens vulgata var. schizantha & Tall Bur-marigold & G5T? & SU & & $\mathrm{W}$ \\
\hline Botrychium minganense & Mingan Island Moonwort & G4 & S3 & & \\
\hline Botrychium montanum & Mountain Moonwort & G3 & S3 & $\mathrm{S}$ & \\
\hline Botrychium simplex & Least Grape-fern & G5 & SU & & \\
\hline Botrychium $x$ watertonense & Waterton Moonwort & HYB & HYB & & \\
\hline Bouteloua hirsuta & Hairy Gramma & G5 & SU & & \\
\hline Calamagrostis koelerioides & Dense Pine Reedgrass & G5 & SA? & & \\
\hline Calamagrostis scopulorum & Ditch Reedgrass & G5 & SU & & \\
\hline Calamagrostis tweedyi & Cascade Reedgrass & G3 & S3 & & \\
\hline Camissonia tanacetifolia & Tansy-leaf Suncup & G5 & SR & & \\
\hline Cardamine rupicola & Cliff Toothwort & G3 & S3 & & \\
\hline Carex aenea & Bronze Sedge & G5 & SU & & \\
\hline Carex bigelowii & Bigelow Sedge & G5 & SR & & \\
\hline Carex brunnescens & Brownish Sedge & G5 & SR & & \\
\hline Carex ebenea & Ebony Sedge & G5 & SU & & $\mathrm{W}$ \\
\hline Carex eburnea & Ivory Sedge & G5 & S3 & & \\
\hline Carex jonesii & Jones' Sedge & G5 & SU & & \\
\hline Carex livida & Pale Sedge & G5 & $\mathrm{S} 3$ & $\mathrm{~S}$ & \\
\hline Carex luzulina var. atropurpurea & Black and Purple Sedge & G5T3 & SU & & \\
\hline
\end{tabular}




\begin{tabular}{|c|c|c|c|c|c|}
\hline Scientific Name & Common Name & $\begin{array}{l}\text { Global } \\
\text { Rank }\end{array}$ & $\begin{array}{l}\text { State } \\
\text { Rank }\end{array}$ & $\begin{array}{c}\text { FS } \\
\text { Status }\end{array}$ & $\begin{array}{l}\text { BLM } \\
\text { Status } \\
\end{array}$ \\
\hline Carex microglochin & False Uncinia Sedge & G5? & SU & & \\
\hline Carex misandra & Short-leaf Sedge & G5 & S3 & & \\
\hline Carex nelsonii & Nelson's Sedge & G3 & SU & & \\
\hline Carex neurophora & Alpine Nerved Sedge & G4 & S3 & & \\
\hline Carex paupercula (C. magellanica) & Poor Sedge & G5 & S3 & $\mathrm{S}$ & \\
\hline Carex pelocarpa & Dusky-seed Sedge & G4G5 & SR & & \\
\hline Carex straminiformis & Mt. Shasta Sedge & G5 & SR & & \\
\hline Carex tenera & Soft Sedge & G5 & SU & & \\
\hline Carex torreyi & Torrey's Sedge & G4 & S3 & & $\mathrm{W}$ \\
\hline Castilleja crista-galli & Greater Red Indian Paintbrush & G4? & SU & & \\
\hline Castilleja gracillima & Slender Indian Paintbrush & G3G4 & SU & $\mathrm{S}$ & $\mathrm{W}$ \\
\hline Castilleja nivea & Snow Indian Paintbrush & G3 & SU & & \\
\hline Castilleja pilosa var. longispica & Parrot-head Indian Paintbrush & G4?T4 & $\mathrm{S} 3$ & $\mathrm{~S}$ & $\mathrm{~W}$ \\
\hline Castilleja rustica (C. flava var. rustica) & Rustic Indian Paintbrush & G4G5T3T4 & S3 & & \\
\hline Chrysosplenium tetrandrum & Northern Golden-carpet & G5 & S3 & & \\
\hline Chrysothamnus linifolius & Lineleaf Rabbitbrush & G5 & S3 & & $\mathrm{W}$ \\
\hline Cirsium pulcherrimum & Wyoming Thistle & G5 & SR & & \\
\hline Claytonia cordifolia & Heart-leaf Springbeauty & G5 & SU & & \\
\hline Collomia debilis var. camporum & Flexible Alpine Collomia & G5T2 & SU & & \\
\hline Conimitella williamsii & Williams' Conimitella & G3? & SU & & \\
\hline Cordylanthus capitatus & Yakima Bird's-beak & G4 & $\mathrm{SR}$ & & \\
\hline Cryptogramma cascadensis & Cascade Rockbrake & G5 & SU & & \\
\hline Cuscuta pentagona var. pentagona & Field Dodder & G5T5 & SU & & \\
\hline $\begin{array}{l}\text { Cymopterus hendersonii } \\
\quad \text { (Pteryxia hendersonii) }\end{array}$ & Henderson's Pteryxia & G5 & $\mathrm{S} 3$ & & \\
\hline Cypripedium $x$ columbianum & Lady’s Slipper (Hybrid) & HYB & HYB & & \\
\hline Delphinium bicolor ssp. calcicola & Limestone Larkspur & G4G5T3 & S3 & & \\
\hline Delphinium burkei & Meadow Larkspur & G4 & SU & & \\
\hline Delphinium geyeri & Geyer's Larkspur & G5 & SU & & \\
\hline Delphinium glaucescens & Electric Peak Larkspur & G3? & SU & & \\
\hline Draba daviesiae & Bitterroot Draba & G3 & $\mathrm{S} 3$ & & \\
\hline Draba lonchocarpa var. exigua & Lance-pod Whitlowgrass & G4T3? & SU & & \\
\hline Echinacea angustifolia & Narrow-leaved Purple Coneflower & $\mathrm{r} \quad \mathrm{G} 4$ & SU & & \\
\hline Elatine americana & American Waterwort & G4 & SU & & \\
\hline Elatine brachysperma & Short-Seeded Waterwort & G5 & SU & & \\
\hline Elatine californica & California Waterwort & G5 & SU & & \\
\hline Eleocharis atropurpurea & Purple Spikerush & G4G5 & SU & & \\
\hline Eleocharis bella & Delicate Spikerush & G5 & SU & & \\
\hline Eleocharis flavescens & Pale Spikerush & G5 & $\mathrm{SR}$ & & \\
\hline Eleocharis xyridiformis & Creeping Spikerush & G4Q & SU & & \\
\hline Equisetum $x$ ferrissii & Horsetail (Hybrid) & HYB & HYB & & \\
\hline Equisetum $x$ trachyodon & Horsetail (Hybrid) & HYB & HYB & & \\
\hline Erigeron allocotus & Big Horn Fleabane & G3 & S3 & & \\
\hline Erigeron coulteri & Coulter Fleabane & G5 & SU & & \\
\hline Erigeron flabellifolius & Fan-leaved Fleabane & G3 & $\mathrm{S} 3$ & & \\
\hline Erigeron flagellaris & Running Fleabane & G5 & SU & & \\
\hline Erigeron lanatus & Woolly Fleabane & G3G4 & S3 & & \\
\hline Eriogonum capistratum var. muhlickii & Muhlick's Buckwheat & G4T? & S3 & & \\
\hline Eriogonum chrysocephalum & A Wild Buckwheat & GUQ & SR & & \\
\hline Eriogonum umbellatum var. aureum & A Wild Buckwheat & G5T3T5 & SU & & \\
\hline Eriophorum scheuchzeri & Scheuchzer's Cottongrass & G5 & SR & & \\
\hline Eriophorum viridicarinatum & Green-keeled Cotton-sedge & G5 & $\mathrm{S} 3$ & & \\
\hline Euthamia graminifolia & Flat-top Fragrant-golden-rod & G5 & SR & & \\
\hline Evax prolifera & Big-head Evax & G5 & SU & & \\
\hline
\end{tabular}




\begin{tabular}{|c|c|c|c|c|c|}
\hline Scientific Name & Common Name & $\begin{array}{c}\text { Global } \\
\text { Rank }\end{array}$ & $\begin{array}{l}\text { State } \\
\text { Rank }\end{array}$ & $\begin{array}{c}\text { FS } \\
\text { Status }\end{array}$ & $\begin{array}{c}\text { BLM } \\
\text { Status }\end{array}$ \\
\hline$\overline{\text { Festuca subulata }}$ & Nodding Fescue & G5 & SU & & \\
\hline Floerkea proserpinacoides & False Mermaid-weed & G5 & SU & & \\
\hline Galium cymosum & Pacific Bedstraw & $\mathrm{G} ?$ & SR & & \\
\hline Gentiana aquatica & Wet Meadow Gentian & G4 & S3 & & \\
\hline Gentiana prostrata & Pygmy Gentian & G5 & SU & & \\
\hline Gentianella tenella & Slender Gentian & G4G5 & SU & & \\
\hline Geum canadense & White Avens & G5 & SU & & \\
\hline Geum rossii var. rossii & Ross's Avens & G5T5 & SR & & \\
\hline Gilia inconspicua & Shy Gilia & G5 & S3 & & \\
\hline Gnaphalium purpureum var. purpureum & Purple Cudweed & G5TU & SA? & & \\
\hline Grindelia nana var. integrifolia & Low Gumweed & G5T? & SU & & \\
\hline Halenia deflexa & Spurred Gentian & G5 & $\mathrm{S} 3$ & & \\
\hline Halimolobos virgata & Twiggy Halimolobos & G4 & S3 & & $\mathrm{W}$ \\
\hline $\begin{array}{l}\text { Haplopappus multicaulis } \\
\quad \text { (Oonopsis multicaulis) }\end{array}$ & Many-stem Goldenweed & G4 & S3 & & \\
\hline Helenium hoopesii & Orange Sneezeweed & G5 & SU & & \\
\hline Hesperochiron californicus & California Hesperochiron & G4G5 & SU & & \\
\hline Heterotheca villosa var. depressa & Low Golden Aster & G5T3 & SR & & \\
\hline Hierochloe alpina & Alpine Sweet Grass & G5 & SR & & \\
\hline Hierochloe odorata & Sweet Grass & G4G5 & $\mathrm{S} 3$ & & \\
\hline Horkelia fusca & Pine Woods Horkelia & G5 & SR & & \\
\hline Huperzia haleakalae & Haleakalae Fir-clubmoss & G4G5 & SU & & \\
\hline Hymenopappus filifolius var. idahoensis & Idaho Hymenopappus & G5T3 & SU & & \\
\hline Hymenopappus filifolius var. luteus & Yellowish Hymenopappus & G5T3T5 & SU & & \\
\hline Impatiens aurella & Pale-yellow Jewel-weed & G4? & SU & & \\
\hline Impatiens ecalcarata & Spurless Touch-me-not & G3G4 & $\mathrm{S} 3$ & & \\
\hline Ipomopsis congesta ssp. pseudotypica & Wyoming Ipomopsis & G5T3? & SU & & \\
\hline Isoetes lacustris & Western Quillwort & G5 & SR & & \\
\hline Isoetes nuttallii & Blackfoot Quillwort & G4? & $\mathrm{SR}$ & & \\
\hline Juncus interior & Inland Rush & G4G5 & SR & & \\
\hline Juncus triglumis & Three-flowered Rush & G5 & SU & & \\
\hline Juncus tweedyi & Tweedy's Rush & G3Q & SU & & \\
\hline Juncus vaseyi & Vasey Rush & G5? & SU & & \\
\hline Lappula cenchrusoides & Great Plains Stickseed & G4 & SU & & \\
\hline Lemna minuta & Least Duckweed & G4 & SU & & \\
\hline Lemna valdiviana & Pale Duckweed & G5 & SU & & \\
\hline Lesquerella klausii & Divide Bladderpod & G3 & S3 & & $\mathrm{W}$ \\
\hline Ligusticum verticillatum & Idaho Lovage & G4G5 & SR & & \\
\hline Lilium columbianum & Columbia Lily & G4G5 & SU & & \\
\hline Lilium philadelphicum & Wood Lily & G5 & $\mathrm{S} 3$ & & \\
\hline Lobelia kalmii & Kalm's Lobelia & G5 & SU & & \\
\hline Lomatium farinosum & Coeur D'Alene Desert-parsley & G4G5 & SR & & \\
\hline Lupinus caudatus & Kellogg's Spurred Lupine & G5 & $\mathrm{SR}$ & & \\
\hline Lycopodium sitchense & Alaskan Clubmoss & G5 & S3 & S & \\
\hline Madia minima & Small-headed Tarweed & G4 & S3 & & \\
\hline Mimulus suksdorfii & Suksdorf Monkeyflower & G4 & S3 & & \\
\hline Muhlenbergia montana & Mountain Muhly & G5 & SR & & \\
\hline Myosotis verna & Early Forget-me-not & G5 & $\mathrm{S} 3$ & & \\
\hline Navarretia divaricata & Mountain Navarretia & G5 & SR & & \\
\hline Oenothera elata ssp. hirsutissima & Hairy Primrose & G5T5 & SR & & \\
\hline Oenothera pallida var. idahoensis & Pale Evening-primrose & G5T?Q & SU & & S \\
\hline Oryzopsis contracta & Contracted Indian Ricegrass & G3G4 & $\mathrm{S} 3$ & & W \\
\hline Oxalis stricta & Upright Yellow Wood-sorrel & G5 & SA? & & \\
\hline Oxytropis besseyi var. fallax & Bessey's Locoweed & G5T3 & SU & & \\
\hline
\end{tabular}




\begin{tabular}{|c|c|c|c|c|c|}
\hline Scientific Name & Common Name & $\begin{array}{l}\text { Global } \\
\text { Rank }\end{array}$ & $\begin{array}{l}\text { State } \\
\text { Rank }\end{array}$ & $\begin{array}{c}\text { FS } \\
\text { Status }\end{array}$ & $\begin{array}{l}\text { BLM } \\
\text { Status }\end{array}$ \\
\hline Oxytropis besseyi var. ventosa & Bessey's Locoweed & G5T3? & SU & & \\
\hline Oxytropis lagopus var. conjugens & Rabbit-foot Crazyweed & G4G5T3 & $\mathrm{S} 3$ & & \\
\hline Pedicularis contorta var. rubicunda & Coil-beaked Lousewort & G5T3 & S3 & & \\
\hline Pedicularis oederi & Oeder's Lousewort & G5 & S3 & & \\
\hline Pedicularis pulchella & Mountain Lousewort & G3 & SU & & \\
\hline Pediocactus simpsonii & Simpson's Hedgehog Cactus & G4 & S3 & & \\
\hline Pediomelum cuspidatum & Large-bracted Scurf-pea & G4 & SR & & \\
\hline Penstemon caryi & Cary's Beardtongue & G3 & S3 & & \\
\hline Penstemon flavescens & Yellow Beardtongue & G3 & S3 & & \\
\hline Penstemon montanus var. idahoensis & Idaho Mountain Beardtongue & G4G5T2T3 & SU & & \\
\hline Physalis hederifolia var. comata & Prairie Ground Cherry & G5?T4? & SR & & \\
\hline Physaria saximontana var. dentata & Rocky Mountain Twinpod & G3T3 & S3 & & \\
\hline Physostegia virginiana & False Dragon-head & G5 & SR & & \\
\hline Platyschkuhria integrifolia & Basin Daisy & G5 & S3 & & \\
\hline Poa macroclada & Rydberg Bluegrass & G5 & SR & & \\
\hline Polygonum polygaloides & White-margined Knotweed & G4G5 & SU & & W \\
\hline $\begin{array}{l}\text { Potentilla concinna var. macounii } \\
\quad \text { (P. macounii) }\end{array}$ & Macoun's Early Cinquefoil & G1?Q & SR & & \\
\hline Psilocarphus elatior & Tall Woollyheads & G4Q & SU & & \\
\hline Psoralea hypogaea & Little Indian Breadroot & G5T4 & S3 & & $\mathrm{W}$ \\
\hline \multicolumn{6}{|c|}{ (Pediomelum hypogaeum var. hypogaeum) } \\
\hline Pyrrocoma integrifolia & Entire-leaf Golden-weed & G3? & SU & & \\
\hline Rubus arcticus & Nagoonberry & G5 & SU & & \\
\hline Salix petiolaris & Meadow Willow & G5 & SR & & \\
\hline Saxifraga chrysantha & Golden Saxifrage & G4 & SU & & \\
\hline Saxifraga marshallii & Marshall Saxifrage & G5 & S3 & & \\
\hline Saxifraga subapetala & Yellowstone Saxifrage & G3G4Q & SU & & \\
\hline Saxifraga tolmiei & Alpine Saxifrage & G5 & $\mathrm{S} 3$ & & \\
\hline Scirpus cyperinus & Wool Grass & G5 & S3 & & $\mathrm{W}$ \\
\hline Scirpus pendulus & Pendulous Bulrush & G5 & SA? & & \\
\hline Sedum leibergii & Borsch's Stonecrop & G4? & S3 & & \\
\hline Selaginella densa var. standleyi & Standley's Compact Selaginella & G5T3T5 & SU & & \\
\hline Senecio congestus & Marsh Flea-bane & G5 & SU & & \\
\hline Senecio debilis & Rocky Mountain Ragwort & G4 & S3 & & \\
\hline Senecio eremophilus & Cut-leaved Groundsel & G5 & SU & & \\
\hline Senecio fuscatus & Twice-hairy Butterweed & G4 & S3 & & \\
\hline Senecio hyperborealis & Boreal Groundsel & G5 & SR & & \\
\hline Silene repens & Creeping Catchfly & G5 & SU & & \\
\hline Sparganium androcladum & Branching Bur-reed & G4G5 & SA? & & \\
\hline Sphaeromeria capitata & Rock-tansy & G3 & S3 & & \\
\hline Spiraea x pyramidata & Pyramidal Spiraea & HYB & HYB & & \\
\hline Sporobolus vaginiflorus & Sheathed Dropseed & G5 & SA? & & \\
\hline Stanleya tomentosa & Hairy Prince-plume & G4 & S3 & & \\
\hline Stipa lemmonii & Lemmon's Needle Grass & G5 & SR & & \\
\hline Stipa pinetorum & Pineforest Needle Grass & G4 & SU & & \\
\hline Synthyris canbyi & Mission Mountain Kittentails & G3 & S3 & & \\
\hline Synthyris missurica & Western Mountain Synthyris & G4 & S3 & & \\
\hline Teucrium canadense & American Germander & G5 & SU & & \\
\hline Thelesperma megapotamicum & Hopi-tea & G5 & SA? & & \\
\hline Tillaea aquatica (Crassula aquatica) & Water Pigmy-weed & G5 & SU & & \\
\hline Townsendia leptotes & Slender Townsendia & G4 & SR & & \\
\hline Townsendia nuttallii & Nuttall's Townsend-daisy & G3 & S3 & & W \\
\hline Townsendia spathulata & Sword Townsendia & G3 & S3 & & \\
\hline Tradescantia bracteata & Bracted Spiderwort & G5 & SR & & \\
\hline
\end{tabular}




\section{Common Name}

Missoula County Oats

Oval-leaf Huckleberry

Dakota Vervain

Fascicled Ironweed

Guelder-rose Viburnum

Alpine Marsh Violet

Prairie Violet

Kidney-leaf White Violet

Northern Blue Violet

Spotted Water-meal
State

Rank

HYB

SR

G5

G5

G5T3T5

G5

G5

G5

G5

G5

G5
FS

Status
BLM

Status

Viola septentrionalis

Wolffia punctata

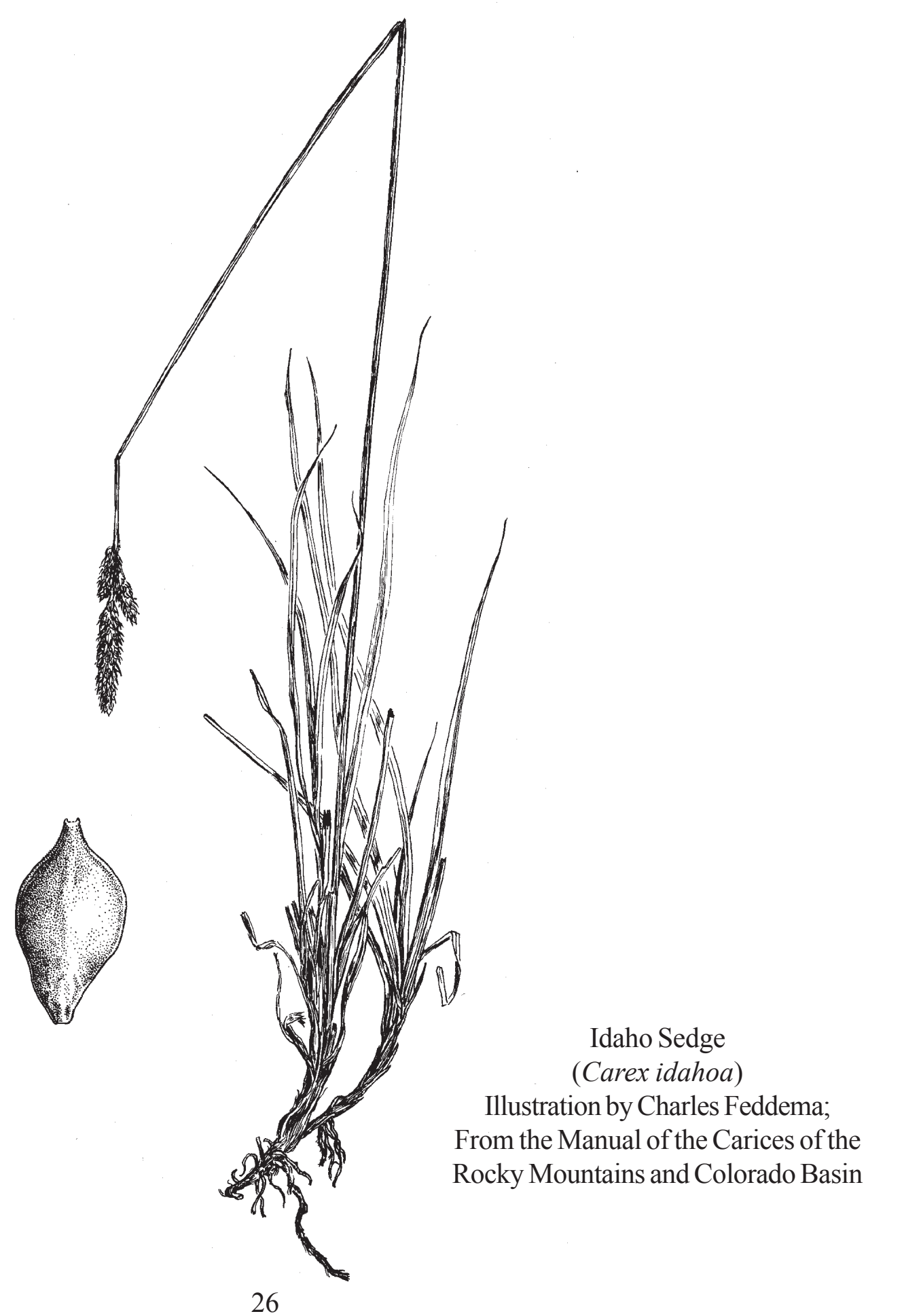




\section{Preliminary Ranks for Nonvascular Plant Species}

\section{INTRODUCTION}

\section{BRYOPHYTES}

The preliminary ranks for rare bryophytes are based on a flora compiled for the U.S. Forest Service by Joe Elliott (1995). This list is unchanged from the 2001 publication, except that some global ranks have been updated. Because nonvascular plants are typically so underrepresented by collection data, the current focus is on species with the fewest documented Montana records (S1 and $\mathrm{SH}$ ) and several species of highly restricted habitats. Principle reference collections for Montana bryophytes are located at the University of Montana (MONTU), Montana State University (MONT), and the U.S. Forest Service's Intermountain Research Station Resource Center Herbarium (MRC) in Missoula. The "counties of distribution" listed here are incomplete because not all Montana collection data has been compiled. Anyone who collects a bryophyte species on this list or one potentially new to the state is encouraged to submit the Species of Concern Survey Form and map, or send a copy of the collection label information to MTNHP.

\section{LICHENS}

The preliminary ranks for rare lichens are based on a Montana lichen flora being compiled by and status recommendations from $\mathrm{McCune}$ and Rosentreter (in progress). Their publication will provide much more information not only on Montana lichens, but also on species of concern and the patterns of rarity exhibited by lichens in Montana. Major reference collections for Montana lichens are located at the University of Montana and Montana State University, in addition to large collections at Oregon State University (OSC), Boise State University (SRP) and the personal herbarium of R.

Rosentreter. Anyone collecting a lichen identified on this list or potentially new to the state is encouraged to submit a MTNHP Survey Form and map, or send a copy of collection label information. Location records for lichens have not yet been entered into the MTNHP databases and are not included below, therefore a county distribution for the species cannot be created as of yet.

\section{Selected References}

Conrad, H.S. and P.L. Redfern. 1979. How to know the mosses and liverworts. Wm. C. Brown Co., Dubuque, Iowa. 302 pp.

Elliott, J.C. Second checklist of Montana mosses (draft). Unpublished report to the U.S. Forest Service. Conservation Biology Research, Helena. 43 pp.

Elliott, J.C. 1995. Rare mosses, rare habitats. Unpublished report for the 1995 Montana Rare Plant Conference. Conservation Biology Research, Helena, MT. $17 \mathrm{pp}$.

Lawton, E. 1971. Keys for the identification of the mosses of the Pacific Northwest (Reprinted from Moss flora of the Pacific Northwest). Hattori Botanical Lab., Nichinan, Japan. 66 pp.

McCune, B. and T. Goward. 1995. Macrolichens of the Northern Rocky Mountains. Mad River Press, Inc. Eureka, CA. 208 pp.

McCune, B. and R. Rosentreter. In progress. Montana Lichens.

Schofield, W.B. 1992. Some common mosses of British Columbia. Royal British Columbia Museum, Victoria, B.C. Canada. 394 pp.

Vitt, D.H., J.E. Marsh and R.B. Bovey. 1988. Mosses, lichens and ferns of northwest North America. Lone Pine Publishing, Edmonton, Alberta, Canada. $296 \mathrm{pp}$. 


\section{Bryophytes}

Scientific Name

Aloina brevirostris

Amblyodon dealbatus

Andreaea blyttii

Barbula eustegia

Brachythecium reflexum

Brachythecium turgidum

Bryum calobryoides

Bryum calophyllum

Bryum dichotomum

Bryum lonchocaulon

Bryum pallens

Bryum schleicheri

Callicladium haldanianum

Calliergon richardsonii

Calliergon trifarium

Calliergonella cuspidata

Catoscopium nigritum

Cinclidium stygium

Crumia latifolia

Cynodontium tenellum

Dendroalsia abietina

Desmatodon cernuus

Desmatodon heimii

Dichodontium olympicum

Dicranella grevilleana

Dicranella heteromalla

Dicranoweisia cirrata

Dicranum acutifolium

Dicranum fragilifolium

Dicranum spadiceum

Didymodon fallax var. reflexus

Didymodon rigidulus var. gracilis

Didymodon vinealis var. brachyphyllus

Distichium inclinatum

Ditrichum ambiguum

Drepanocladus cardotti

Entosthodon rubiginosus

Eucladium verticillatum

Eurhynchium pulchellum var. barnesii

Fabronia pusilla

Fissidens fontanus

Funaria americana

Grimmia brittoniae

Grimmia incurva

Grimmia mollis

Hamatocaulis vernicosus

Herzogiella striatella

Herzogiella turfacea

Hygroamblystegium noterophilum

Hygrohypnum cochlearifolium

Hypnum callichroum

Kiaeria blyttii

Kiaeria falcata

\section{Family}

Pottiaceae

Meesiaceae

Andreaeaceae

Meteoriaceae

Brachytheciaceae

Brachytheciaceae

Bryaceae

Bryaceae

Bryaceae

Bryaceae

Bryaceae

Bryaceae

Hypnaceae

Amblystegiaceae

Amblystegiaceae

Amblystegiaceae

Catoscopiaceae

Mniaceae

Pottiaceae

Dicranaceae

Cryphaeaceae

Pottiaceae

Pottiaceae

Dicranaceae

Dicranaceae

Dicranaceae

Dicranaceae

Dicranaceae

Dicranaceae

Dicranaceae

Pottiaceae

Pottiaceae

Pottiaceae

Ditrichaceae

Ditrichaceae

Amblystegiaceae

Funariaceae

Pottiaceae

Brachytheciaceae

Fabroniaceae

Fissidentaceae

Funariaceae

Grimmiaceae

Grimmiaceae

Grimmiaceae

Amblystegiaceae

Hypnaceae

Hypnaceae

Amblystegiaceae

Amblystegiaceae

Hypnaceae

Dicranaceae

Dicranaceae
Global
Rank

G3G5

G3G5

G5

G3?

G4G5

G4

G3

G5?

G5?

G5?

G4G5

G5?

G5

G4

G4

G5

G4G5

G5

G3

G3G5Q

G4

G3G5

G5

G3G5

G2G4

G5?

G4

G5?

G4G5

G5?

G5T5?

G5T5?

G5T?

G4G5

G4?

G1G2

G1G3

G4

G5T?

G5?

G5

G2G3

G1

G4G5

G3G5

G5

G4G5

G4G5

G4

G4

G5?

G5

G5
State FS County Rank Status Distribution

S 1

$\mathrm{SH}$

S 1

S 1

S 1

S 1

S 1

$\mathrm{SH}$

S1

S 1

S1

S1

$\mathrm{SH}$

S 1

S1

S1

S1

S1

S 1

SH

$\mathrm{SH}$

$\mathrm{SH}$

S 1

S1

S1

S1

S1

S1

S1

S1

S1

S1

S1

S1

$\mathrm{SH}$

$\mathrm{SH}$

$\mathrm{SH}$

S 1

S 1

S 1

S 1

$\mathrm{SH}$

S1

S 1

S1

S1

S1

S1

S 1

S 1

S1

S1

S1
FLAT, LINC

FLAT

SAND

N/A

FLAT, LINC

N/A

FLAT, LAKE

N/A

RAVA

N/A

$\mathrm{N} / \mathrm{A}$

GLAC

$\mathrm{N} / \mathrm{A}$

GLAC

GLAC

FLAT

N/A

N/A

N/A

N/A

N/A

N/A

RAVA

N/A

FLAT, GLAC

FLAT, GLAC

PARK

RAVA

FLAT, GLAC, LAKE

N/A

$\mathrm{N} / \mathrm{A}$

$\mathrm{N} / \mathrm{A}$

RAVA

GLAC

FLAT

N/A

CASC

GRAN, POWE

LAKE, RAVA, SAND

MADI

GRAN

CASC, MEAG

S FLAT, SAND

RAVA

FLAT

N/A

$\mathrm{N} / \mathrm{A}$

N/A

$\mathrm{N} / \mathrm{A}$

LINC

N/A

FLAT

N/A 
Bryophytes (cont'd)

\section{Scientific Name}

Kiaeria starkei

Leucolepis acanthoneuron

Meesia longiseta

Meesia triquetra

Meesia uliginosa

Mielichhoferia macrocarpa

Myurella tenerrima

Neckera douglasii

Neckera pennata

Oligotrichum aligerum

Orthotrichum praemorsum

Oxystegus tenuirostris

Paludella squarrosa

Paraleucobryum enerve

Paraleucobryum longifolium

Phascum cuspidatum

Physcomitrium hookeri

Physcomitrium pyriforme

Plagiobryum demissum

Plagiobryum zieri

Platyhypnidium riparioides

Pohlia drummondii

Pohlia obtusifolia

Pohlia vexans

Porotrichum bigelovii

Pseudocalliergon turgescens

Pseudocrossidium obtusulum

Pterygoneurum lamellatum

Ptychomitrium gardneri

Racomitrium aquaticum

Racomitrium brevipes

Racomitrium pygmaeum

Sarmenthypnum sarmentosum

Schistostega pennata

Scorpidium scorpioides

Seligeria donniana

Sphagnum centrale

Sphagnum compactum

Sphagnum contortum

Sphagnum fallax

Sphagnum fimbriatum

Sphagnum girgensohnii

Sphagnum magellanicum

Sphagnum mendocinum

Sphagnum platyphyllum

Sphagnum riparium

Sphagnum subnitens

Sphagnum wulfianum

Stegonia latifolia

Tayloria acuminata

Tayloria lingulata $\begin{array}{cc}\text { Gamily } & \text { Global } \\ \text { Rank }\end{array}$

Dicranaceae

Mniaceae

Meesiaceae

Meesiaceae

Meesiaceae

Bryaceae

Pterigynandraceae

Neckeraceae

Neckeraceae

Polytrichaceae

Orthotrichaceae

Pottiaceae

Meesiaceae

Dicranaceae

Dicranaceae

Pottiaceae

Funariaceae

Funariaceae

Bryaceae

Bryaceae

Brachytheciaceae

Bryaceae

Bryaceae

Bryaceae

Thamnobryaceae

Amblystegiaceae

Pottiaceae

Pottiaceae

Ptychomitriaceae

Grimmiaceae

Grimmiaceae

Grimmiaceae

Amblystegiaceae

Schistostegaceae

Amblystegiaceae

Seligeriaceae

Sphagnaceae

Sphagnaceae

Sphagnaceae

Sphagnaceae

Sphagnaceae

Sphagnaceae

Sphagnaceae

Sphagnaceae

Sphagnaceae

Sphagnaceae

Sphagnaceae

Sphagnaceae

Pottiaceae

Splachnaceae

Splachnaceae

G5

G4

G4?

G5

G4

G2

G4

G5

G5

GU

G5

G5?

G5

G5

G5

G5

G4

GU

G4

GU

GU

G4

G5

G5

G5

G5

G5

G5

G5

G4

G5

G5

G5

G5
State FS County

Rank Status Distribution

G3G4

G3G5

G2G4

G3G5

G3G4

G3G4

G2G4

G3G5

G3G5

G3G5

G2G4

G4G5

G4G5

G4G5

G4G5

G3G4

G3G5

S1

S 1

S 1

S2

S1

S 1

S1

S1

S1

S1

S1

SH

S1

S1

S1

S1

SH

SU

S1

S 1

S1

S1

$\mathrm{S} 1$

$\mathrm{SH}$

S 1

$\mathrm{S} 1$

S1

S1

S1

S1

S1

S1

S 1

S1

S2

S1

S 1

S1

S1

S1

S1

S1

S1

S1

S1

S 1

S 1

S1

S1

SH

S 1
GLAC

LINC

N/A

S CARB, FLAT, GLAC, LAKE

LINC, RAVA, TETO

FLAT, GLAC

N/A

GLAC

FLAT, LAKE

N/A

LINC

RAVA

N/A

CARB, GLAC

GLAC

FLAT

RAVA

N/A

RAVA

N/A

N/A

LINC

N/A

S N/A

MEAG

RAVA

FLAT

RAVA

N/A

N/A

N/A

N/A

LINC

N/A

N/A

S FLAT, GLAC, LAKE, LEWI

LINC, MISS, TETO

N/A

FLAT

GRAN

FLAT

N/A

LEWI

N/A

FLAT, MISS, RAVA

MISS

N/A

MISS

N/A

N/A

N/A

N/A

GLAC 
Bryophytes (cont'd)

Scientific Name

Tayloria serrata

Thamnobryum neckeroides

Tortula bartramii

Tortula muralis

Tortula norvegica

Tortula papillosa

Warnstorfia exannulata

\section{Family}

Splachnaceae

Thamnobryaceae

Pottiaceae

Pottiaceae

Pottiaceae

Pottiaceae

Amblystegiaceae

\section{Global Rank}

G4

GU

G2G4

G5

G5

G5

G5
State FS County Rank Status

S1

SH

$\mathrm{S} 1$

$\mathrm{S} 1$

S 1

$\mathrm{S} 1$

S 1
Distribution

MADI

N/A

RAVA

RAVA

GLAC, LAKE, MADI

RAVA, SAND

N/A

\section{Lichens}

\section{Scientific Name}

Acarospora scabrida

Adelolecia pilati

Alectoria nigricans

Alectoria vexillifera

Arctoparmelia subcentrifuga

Aspicilia arctica

Aspicilia fruticulosa

Aspicilia myrinii

Bacidia auerswaldii

Bacidia granosa

Bacidia vermifera

Bryonora castanea

Bryoria friabilis

Bryoria implexa

Bryoria nadvornikiana

Bryoria simplicior

Bryoria tortuosa

Buellia badia

Cetraria subalpina

Chaenotheca cinerea

Chaenotheca subroscida

Cladina mitis

Cladina rangiferina

Cladonia botrytes

Cladonia digitata

Cladonia transcendens

Cladonia uncialis

Cliostomum leprosum

Collema curtisporum

Collema polycarpon

Cornicularia normoerica

Cyphelium karelicum

Dactylina madreporiformis

Dactylina ramulosa

Dimelaena thysanota

Diploschistes diacapsis

Endocarpon pulvinatum

Endocarpon tortuosum

Euopsis granatina

Evernia divaricata

Evernia prunastri

(c) 2003 Montana Natural Heritage Program

\begin{tabular}{|c|c|c|c|}
\hline Family & $\begin{array}{c}\text { Global } \\
\text { Rank }\end{array}$ & $\begin{array}{l}\text { State } \\
\text { Rank }\end{array}$ & $\begin{array}{c}\text { FS } \\
\text { Status }\end{array}$ \\
\hline Acarosporaceae & G3G5 & $\mathrm{S} 1$ & SENSITIVE \\
\hline Bacidiaceae & G2G4 & $\mathrm{S} 1$ & \\
\hline Alectoriaceae & G5 & $\mathrm{S} 1$ & \\
\hline Alectoriaceae & $\mathrm{G} ?$ & $\mathrm{~S} 2$ & \\
\hline Parmeliaceae & $\mathrm{G} ?$ & $\mathrm{~S} 1$ & \\
\hline Hymeneliaceae & G2G4 & $\mathrm{S} 1$ & \\
\hline Hymeneliaceae & G3 & $\mathrm{S} 1$ & \\
\hline Hymeneliaceae & G5 & $\mathrm{S} 1$ & \\
\hline Bacidiaceae & $\mathrm{G} ?$ & $\mathrm{~S} 1$ & \\
\hline Bacidiaceae & $\mathrm{G} ?$ & $\mathrm{~S} 1$ & \\
\hline Bacidiaceae & G2G4 & $\mathrm{S} 1$ & \\
\hline Lecanoraceae & G3G5 & $\mathrm{S} 1$ & \\
\hline Parmeliaceae & G3 & $\mathrm{S} 2$ & \\
\hline Parmeliaceae & $\mathrm{G} ?$ & $\mathrm{~S} 1$ & \\
\hline Parmeliaceae & $\mathrm{G} ?$ & $\mathrm{~S} 1$ & \\
\hline Parmeliaceae & $\mathrm{G} ?$ & $\mathrm{~S} 2$ & \\
\hline Parmeliaceae & $\mathrm{G} ?$ & $\mathrm{~S} 1$ & \\
\hline Physciaceae & G3? & $\mathrm{S} 1$ & \\
\hline Parmeliaceae & G3? & $\mathrm{S} 2$ & SENSITIVE \\
\hline Coniocybaceae & $\mathrm{G} ?$ & $\mathrm{~S} 1$ & \\
\hline Coniocybaceae & $\mathrm{G} ?$ & $\mathrm{~S} 1$ & \\
\hline Cladoniaceae & G5 & $\mathrm{S} 1$ & \\
\hline Cladoniaceae & G5 & $\mathrm{S} 1$ & \\
\hline Cladoniaceae & G5 & $\mathrm{S} 1$ & \\
\hline Cladoniaceae & G3G5 & $\mathrm{S} 1$ & \\
\hline Cladoniaceae & G5 & $\mathrm{S} 1$ & \\
\hline Cladoniaceae & G3G5 & $\mathrm{S} 1$ & \\
\hline Bacidiaceae & $\mathrm{G} ?$ & $\mathrm{~S} 1$ & \\
\hline Collemataceae & G3 & $\mathrm{S} 2$ & SENSITIVE \\
\hline Collemataceae & $\mathrm{G} ?$ & S2 & \\
\hline Usneaceae & G3G5 & $\mathrm{S} 1$ & \\
\hline Caliciaceae & $\mathrm{G} ?$ & $\mathrm{~S} 1$ & \\
\hline Parmeliaceae & $\mathrm{G} ?$ & $\mathrm{~S} 2$ & \\
\hline Parmeliaceae & $\mathrm{G} ?$ & $\mathrm{~S} 2$ & \\
\hline Buelliaceae & $\mathrm{G} ?$ & $\mathrm{~S} 1$ & \\
\hline Thelotremataceae & $\mathrm{G} ?$ & $\mathrm{~S} 1$ & \\
\hline Verrucariaceae & $\mathrm{G} ?$ & $\mathrm{~S} 2$ & \\
\hline Verrucariaceae & $\mathrm{G} ?$ & $\mathrm{~S} 2$ & \\
\hline Lichinaceae & $\mathrm{G} ?$ & $\mathrm{~S} 1$ & \\
\hline Parmeliaceae & $\mathrm{G} ?$ & $\mathrm{~S} 1$ & \\
\hline Parmeliaceae & G5 & S3 & \\
\hline
\end{tabular}


Lichens (cont'd)

Scientific Name

Farnoldia micropsis

Cetraria cucullata (Flavocetraria cucullata)

Flavopunctelia flaventior

Gyalecta foveolaris

Gyalecta jenensis

Hypogymnia enteromorpha

Hypogymnia inactiva

Imshaugia aleurites

Japewia tornoensis

Lecania fuscella

Lecanora beringii

Lecanora umbrosa

Lecidea dolodes

Lecidia myriocarpoides

Lecidea paddensis

Lecidella effugiens

Leptogium minutissimum

Leptogium tenuissimum

Lobaria hallii

Lobaria linita

Lobaria scrobiculata

Cetraria conmixta (Melanelia commixtra)

Melanelia septentrionalis

Micarea ternaria

Miriquidica garovaglii

Bacidia sabuletorum (Mycobilimbia sabuletorum)

Neofuscelia loxodes

Neofuscelia subhosseana

Nodobryoria subdivergens (Bryoria subdivergens)

Ophioparma rubricosa

Parmelia fraudans

Parmelia omphalodes

Parmeliella triptophylla

Peltula polyspora

Pertusaria amara

Pertusaria saximontana

Pertusaria sommerfeltii

Phaeophyscia ciliata

Phaeophyscia kairamoi

Phaeorrhiza sareptana

Physcia semipinnata

Platismatia stenophylla

Pseudocyphellaria anomala

Psora rubiformis

Psorotichia nigra

Psorotichia schaereri

Ramalina farinacea

Ramalina obtusata

Ramalina pollinaria

Ramalina thrausta

Rhizocarpon hochstetteri

Rhizocarpon intermediellum

Rhizocarpon polycarpum
Family

Porpidiaceae

Parmeliaceae

Parmeliaceae

Gyalectaceae

Gyalectaceae

Parmeliaceae

Parmeliaceae

Parmeliaceae

Bacidiaceae

Bacidiaceae

Lecanoraceae

Lecanoraceae

Lecideaceae

Lecideaceae

Lecideaceae

Lecanoraceae

Collemataceae

Collemataceae

Lobariaceae

Lobariaceae

Lobariaceae

Pareliaceae

Parmeliaceae

Micareaceae

Lecanoraceae

Porpidiaceae

Parmeliaceae

Parmeliaceae

Parmeliaceae

Ophioparmaceae

Parmeliaceae

Parmeliaceae

Pannariaceae

Peltulaceae

Pertusariaceae

Pertusariaceae

Pertusariaceae

Physciaceae

Physciaceae

Physciaceae

Physciaceae

Parmeliaceae

Lobariaceae

Psoraceae

Lichinaceae

Lichinaceae

Ramalinaceae

Ramalinaceae

Ramalinaceae

Ramalinaceae

Rhizocarpaceae

Rhizocarpaceae

Rhizocarpaceae

$\begin{array}{llc}\begin{array}{l}\text { Global } \\ \text { Rank }\end{array} & \begin{array}{c}\text { State } \\ \text { Rank }\end{array} & \text { FS } \\ \text { Status }\end{array}$

G?

G5

G5

$\mathrm{G}$ ?

$\mathrm{G}$ ?

G4

G3

G5

$\mathrm{G}$ ?

$\mathrm{G}$ ?

$\mathrm{G}$ ?

$\mathrm{G}$ ?

$\mathrm{G}$ ?

G

$\mathrm{G}$ ?

$\mathrm{G}$ ?

$\mathrm{G}$ ?

$\mathrm{G}$ ?

G4

G4

G3G4 S1

$\mathrm{G}$ S 1

G3G5 S1

G? $\mathrm{S} 1$

G? $\mathrm{S} 1$

G? $\quad \mathrm{S} 1$

G3G5 S1

G? S2

G2 S2

$\mathrm{G}$ S3

$\mathrm{G}$ S 1

G2G4 S1

$\mathrm{G} ? \quad \mathrm{~S} 1$

$\mathrm{G} ? \quad \mathrm{~S} 1$

G? S1

G? S1

G? $\quad \mathrm{S} 1$

G4G5 S1

G3G4 S1

G? $\quad$ S 1

G5 $\mathrm{S} 1$

G2G4 S1

G2G4 S1

G? $\quad \mathrm{S} 1$

G? S1

G? $\quad \mathrm{S} 1$

G3G5 S2

$\mathrm{G}$ ? $\mathrm{S} 3$

G2G4 S1

G3G5 S3

G? $\mathrm{S} 1$

$\mathrm{G}$ S 1

G? S1
SENSITIVE 
Lichens (cont'd)

\begin{tabular}{llcc} 
Scientific Name & Family & $\begin{array}{c}\text { Global } \\
\text { Rank }\end{array}$ & $\begin{array}{c}\text { State } \\
\text { Rank }\end{array}$ \\
\hline Rhizoplaca haydenii & Lecanoraceae & G? & S1 \\
Solorina bispora & Peltigeraceae & G3G5 & S1 \\
Solorina octospora & Peltigeraceae & G3G5 & S1 \\
Solorina saccata & Peltigeraceae & G3G5 & S2 \\
Solorina spongiosa & Peltigeraceae & G3G5 & S1 \\
Sporastatia polyspora & Acarosporaceae & G? & S1 \\
Stereocaulon paschale & Stereocaulaceae & G5 & S1 \\
Toninia alutacea & Catillariaceae & G? & S1 \\
Toninia candida & Catillariaceae & G? & S2 \\
Toninia ruginosa & Catillariaceae & G? & S2 \\
Cetraria sepincola (Tuckermannopsis sepincola) $)$ & Parmeliaceae & G4 & S2 \\
Umbilicaria havaasii & Umbilicariaceae & G3 & S1 \\
Umbilicaria hirsuta & Umbilicariaceae & G2G4 & S1 \\
Umbilicaria lambii & Umbilicariaceae & G2G4 & S1 \\
Umbilicaria muehlenbergii & Umbilicariaceae & G? & S1 \\
Umbilicaria polyrhiza & Umbilicariaceae & G2G3 & S1 \\
Usnea cavernosa & Parmeliaceae & G3G5 & S1 \\
Verrucaria calkinsiana & Verrucariaceae & G? & S1 \\
Xanthoparmelia angustiphylla & Parmeliaceae & G5 & S1 \\
Xanthoparmelia montanensis & Parmeliaceae & G5 & S1
\end{tabular}

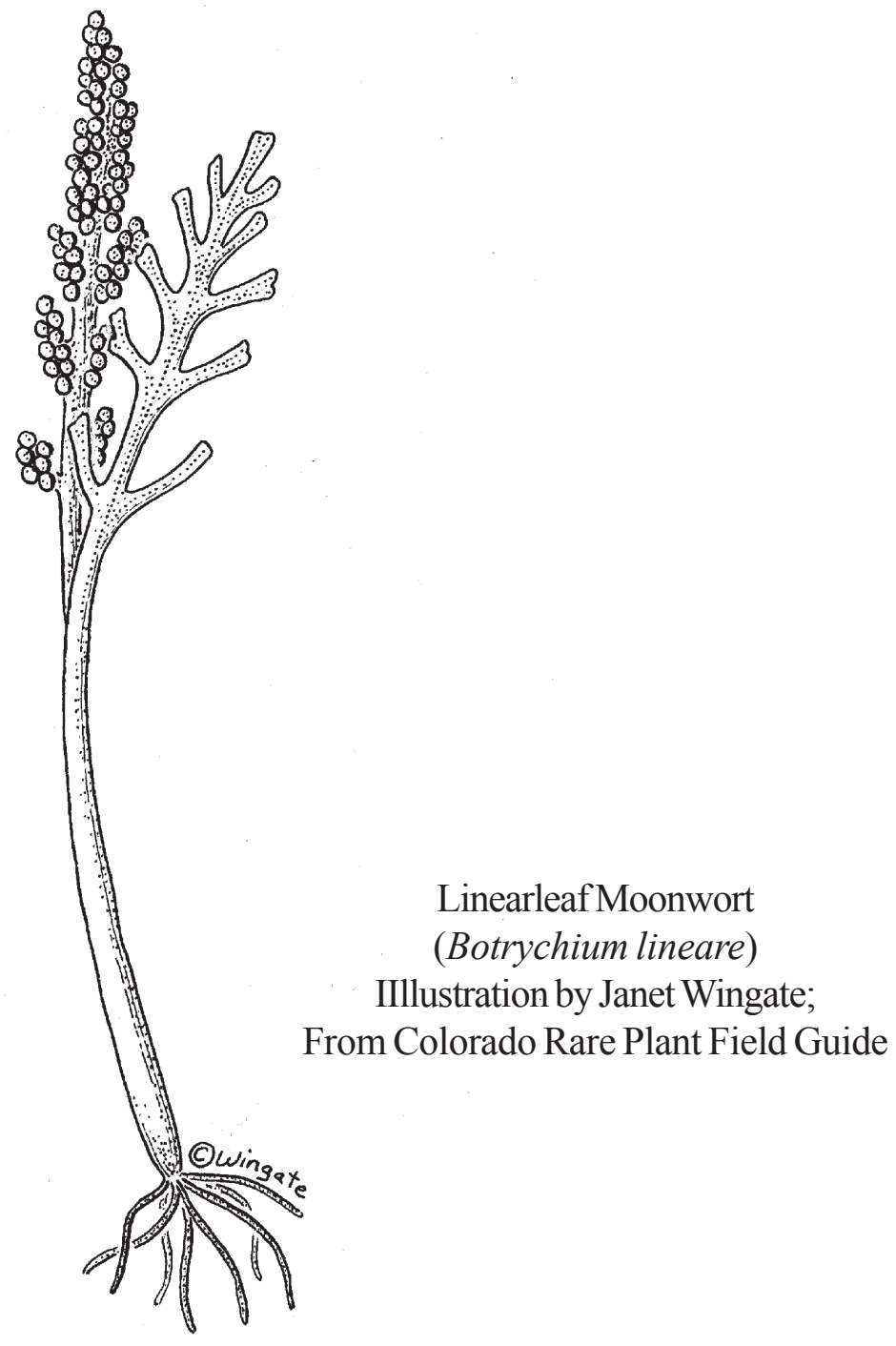




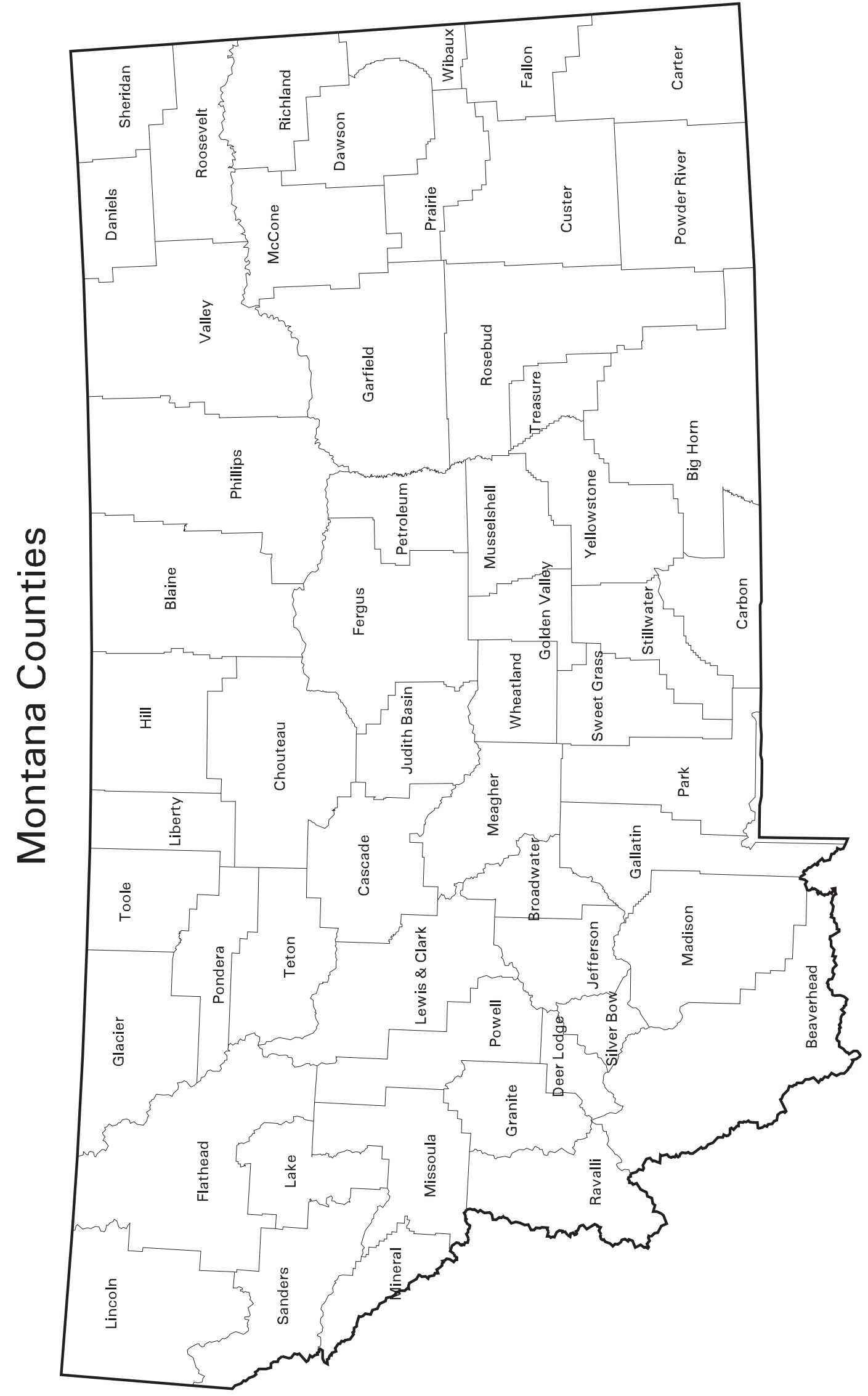




\section{Forest Service Sensitive Plant List}

$\underline{\text { Sensitive - Vascular }}$

Adoxa moschatellina

Agastache cusickii

Allium acuminatum

Allium parvum

Allotropa virgata

Amerorchis rotundifolia

Antennaria densifolia

Aquilegia brevistyla

Arabis fecunda

Asclepias ovalifolia

Astragalus barrii

Astragalus lackschewitzii

Astragalus scaphoides

Athysanus pusillus

Balsamorhiza macrophylla

Bidens beckii

Botrychium ascendens

Botrychium crenulatum

Botrychium hesperium

Botrychium montanum

Botrychium paradoxum

Botrychium pedunculosum

Brasenia schreberi

Carex amplifolia

Carex chordorrhiza

Carex gravida var. gravida

Carex idahoa

Carex livida

Carex paupercula

Carex prairea

Carex rostrata

Carex vaginata

Castilleja covilleana

Castilleja gracillima

Castilleja pilosa var. longispica

Cirsium longistylum

Clarkia rhomboidea

Claytonia arenicola

Cypripedium fasciculatum

Cypripedium parviflorum

Cypripedium passerinum

Drosera anglica

Drosera linearis

Dryopteris cristata

Eleocharis rostellata

Elymus innovatus

Epipactis gigantea

Erigeron asperugineus

Erigeron evermannii

Erigeron lackschewitzii

Eriophorum gracile

Eupatorium occidentale

Gentianopsis macounii

Gentianopsis simplex

Glossopetalon nevadense

Goodyera repens

Grindelia howellii

Halimolobos perplexa var. lemhiensis

Haplopappus aberrans

Haplopappus carthamoides var. subsquarrosus

\section{Sensitive (Con't)}

Haplopappus macronema var. macronema

Heteranthera dubia

Heterocodon rariflorum

Howellia aquatilis - LT

Idahoa scapigera

Juncus hallii

Kalmia polifolia

Lathyrus bijugatus

Lesquerella humilis

Lesquerella paysonii

Lesquerella pulchella

Liparis loeselii

Lomatium geyeri

Lomatogonium rotatum

Lycopodium dendroideum

Lycopodium inundatum

Lycopodium sitchense

Mertensia bella

Mimulus patulus

Mimulus primuloides

Ophioglossum pusillum

Orogenia fusiformis

Oxytropis campestris var. columbiana

Oxytropis podocarpa

Penstemon lemhiensis

Penstemon payettensis

Petasites frigidus var nivalis

Phlox kelseyi var missoulensis

Polygonum douglasii ssp. austinae

Potamogeton obtusifolius

Potentilla quinquefolia

Psilocarphus brevissimus var. brevissimus

Ranunculus jovis

Salix barrattiana

Salix wolfii var. wolfii

Saussurea weberi

Saxifraga tempestiva

Scheuchzeria palustris

Scirpus cespitosus

Scirpus subterminalis

Shoshonea pulvinata

Thalictrum alpinum

Thelypteris phegopteris

Thlaspi parviflorum

Trifolium eriocephalum ssp. arcuatum

Trifolium gymnocarpon

Utricularia intermedia

Veratrum californicum

Viola renifolia

Waldsteinia idahoensis

Sensitive - Nonvascular

Bryoria subdivergens

Cetraria subalpina

Collema curtisporum

Grimmia brittoniae

Meesia triquetra

Scorpidium scorpioides

\section{Bureau of Land Management Sensitive Plant List}

$\underline{\text { Sensitive }}$

Agastache cusickii

Arabis fecunda

Astragalus ceramicus var. apus

Astragalus geyeri

Astragalus geyeri var. geyeri

Astragalus scaphoides

Astragalus terminalis

Camissonia andina

Camissonia parvula

Carex crawei

Carex idahoa

Cryptantha scoparia

Elymus flavescens

Eriogonum salsuginosum

Lesquerella carinata var. languida

Lesquerella lesicii

Lesquerella pulchella

Lomatium attenuatum

Malacothrix torreyi

Nama densum

Oenothera pallida var. idahoensis

Penstemon lemhiensis

Penstemon whippleanus

Quercus macrocarpa

Shoshonea pulvinata

Sphaeromeria argentea

Taraxacum eriophorum

Thalictrum alpinum

Thelypodium paniculatum

\section{Watch}

Adoxa moschatellina

Ambrosia acanthicarpa

Amerorchis rotundifolia

Arabis demissa var. languida

Asclepias ovalifolia

Asclepias stenophylla

Astragalus aretioides

Astragalus barrii

Astragalus convallarius var. convallarius

Astragalus oreganus

Astragalus racemosus var. longisetus

Atriplex truncata

Bacopa rotundifolia

Balsamorhiza macrophylla

Bidens comosa

Bidens vulgata var. schizantha

Carex eburnea

Carex gravida var. gravida

Carex multicostata

Carex occidentalis

Carex torreyi

Castilleja gracillima

Castilleja pilosa var. longispica

Ceanothus herbaceus var. pubescens

Celastrus scandens

Centunculus minimus

Cercocarpus montanus var. glaber

Chenopodium subglabrum

Chrysothamnus linifolius

Cleome lutea

Conioselinum scopulorum

Cryptantha fendleri

Cryptantha humilis

Cyperus acuminatus
Watch (Con't)

Cyperus schweinitzii

Cypripedium parviflorum

Dalea enneandra

Dalea villosa var. villosa

Dichanthelium oligosanthes var. scribnerianum

Downingia laeta

Draba globosa

Eleocharis rostellata

Elodea longivaginata

Epipactis gigantea

Erigeron asperugineus

Erigeron formosissimus var. viscidus

Eupatorium maculatum var. bruneri

Eustoma grandiflorum

Gentianopsis macounii

Gentianopsis simplex

Grayia spinosa

Halimolobos virgata

Haplopappus carthamoides var. subsquarrosus

Hutchinsia procumbens

Leptodactylon caespitosum

Lesquerella klausii

Lomatium nuttallii

Lomatogonium rotatum

Machaeranthera commixta

Mentzelia nuda

Mentzelia pumila

Mirabilis hirsuta

Najas guadalupensis

Nuttallanthus texanus

Oryzopsis contracta

Penstemon angustifolius

Phacelia incana

Phacelia scopulina

Phacelia thermalis

Phlox andicola

Plagiobothrys leptocladus

Poa curta

Polygonum polygaloides

Potentilla plattensis

Primula alcalina

Primula incana

Psilocarphus brevissimus var. brevissimus

Psoralea hypogaea

Ranunculus cardiophyllus

Ranunculus pedatifidus

Rorippa calycina

Salix serissima

Scirpus cyperinus

Solidago sparsiflora

Sphenopholis intermedia

Spiranthes diluvialis - LT

Sporobolus asper

Sporobolus neglectus

Stellaria crassifolia

Stellaria jamesiana

Stephanomeria spinosa

Sullivantia hapemanii var. hapemanii

Townsendia condensata

Townsendia florifer

Townsendia nuttallii

Veratrum californicum

Viola renifolia

Wolffia columbiana 

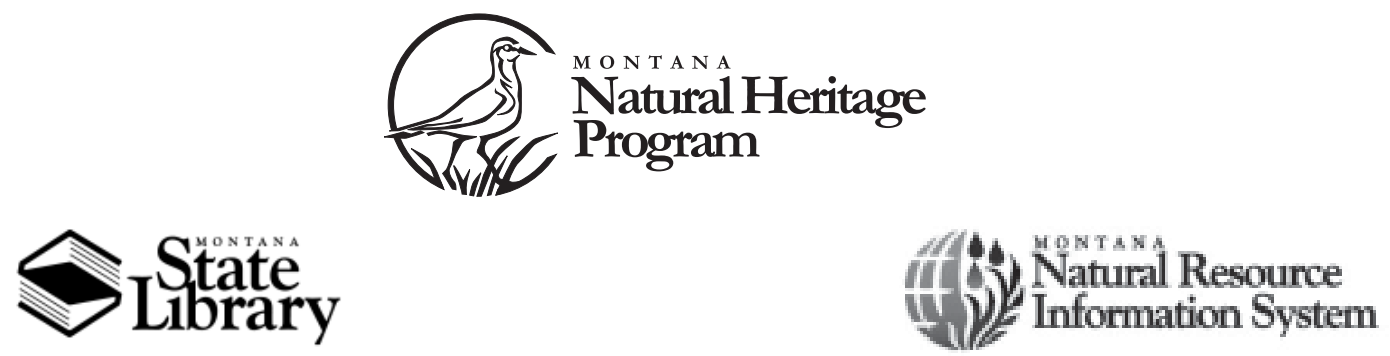